\title{
Push-forwards for Witt groups of schemes
}

\author{
Baptiste Calmès and Jens Hornbostel
}

\begin{abstract}
Using suitable closed symmetric monoidal structures on derived categories of schemes, as well as adjunctions of the type $\left(\mathrm{L} f^{*}, \mathrm{R} f_{*}\right)$ and $\left(\mathrm{R} f_{*}, f^{!}\right)$(i.e. Grothendieck duality theory), we define push-forwards for coherent Witt groups along proper morphisms between separated noetherian schemes. We also establish fundamental theorems for these push-forwards (e.g. base change and projection formula) and provide some computations.
\end{abstract}

Mathematics Subject Classification (2010). 19G12, 19G38, 18F30, 11E81, $19 \mathrm{E} 08$.

Keywords. Witt groups of schemes, push-forward, transfer, Grothendieck duality, quadratic forms.

\section{Introduction}

Push-forwards, also known as transfers or norm maps, exist for many cohomology theories over schemes, e.g. for $K$-theory, (higher) Chow groups and algebraic cobordism. They are undoubtedly a useful tool for understanding and computing those cohomology theories. The present article is about the construction of such pushforward maps for the coherent Witt groups of schemes defined in the seminal work of Balmer $^{1}$. A reader familiar with cohomology theories might think that constructing a push-forward is probably straightforward. He (or she) should be warned: Witt groups are not an oriented cohomology theory. In particular, push-forwards are, in some sense, only conditionally defined. For example, when $X$ and $Y$ are connected noetherian schemes of finite Krull dimension, smooth over a field, the Witt groups depend on a line bundle $L$ used to define the duality and the push-forward takes the form (see Theorem 6.7)

$$
\mathrm{W}^{i+\operatorname{dim} X}\left(X, \omega_{X} \otimes f^{*} L\right) \rightarrow \mathrm{W}^{i+\operatorname{dim} Y}\left(Y, \omega_{Y} \otimes L\right)
$$

\footnotetext{
${ }^{1}$ The modern definition of Witt groups using triangulated categories with dualities [2] can be applied either to the derived category of complexes of locally free sheaves to obtain "locally free" Witt groups or to the derived category of complexes with coherent cohomology to obtain "coherent" Witt groups. As with $K$-theory, it is the latter that is naturally covariant along proper morphisms, as we prove in this article. All schemes considered are over $\mathbb{Z}[1 / 2]$ so that the derived categories involved are $\mathbb{Z}[1 / 2]$-linear and the machinery of triangular Witt groups applies.
} 
where $\omega$ is the canonical bundle (the highest nontrivial exterior power of the cotangent bundle) and $L$ is an arbitrary line bundle on $Y$. In particular, if we pick a line bundle $K$ over $X$, there is no push-forward starting from $\mathrm{W}^{i}(X, K)$ if $K$ is not isomorphic to $\omega_{X} \otimes f^{*} L$ for some $L$ (up to a square $M^{\otimes 2}$, as $\mathrm{W}^{i}(Y, K) \cong \mathrm{W}^{i}\left(Y, K \otimes M^{\otimes 2}\right.$ ) so only the class of $K$ in $\operatorname{Pic}(X) / 2$ really matters). This fundamental difference with oriented cohomology theories, where the push-forward is always possible, significantly changes classical computations, as one sees e.g. in [18], [39], [4]. The groups $\mathrm{W}^{\mathrm{dim} X-i}\left(X, \omega_{X}\right)$ can be considered as a homology theory analogous to a non oriented complex homology theory in topology, but the construction of the push-forward here relies on triangulated monoidal methods.

Besides this article and its precursors on regular schemes [10] and [11], there are already several articles available on the construction of push-forwards in special cases. In [16], Gille defined push-forwards along finite morphisms in the affine case. His approach is quite elementary in the sense that he uses direct computations involving explicit injective resolutions etc. It is useful to get a hand on concrete forms. In [31] and [32], Nenashev adapts the oriented cohomology techniques of Panin and Smirnov to the non-oriented case of locally free Witt groups. He thus obtains push-forwards along projective morphisms between smooth quasi-projective varieties over fields. Still another approach using stable $\mathbb{A}^{1}$-representability of Witt groups can be found in [24]. We understand that there is also some unpublished work of C. Walter on this subject. Our approach is different, and it applies to a much larger class of situations: it uses derived functors and Grothendieck duality, so the dualities that appear are canonical and do not depend on choices as the other constructions mentioned above. If necessary, choices can be made in order to compare our constructions with others in the special cases where the latter are defined. Fundamental properties such as base change are proved in a simple and conceptual way, and we furthermore obtain the full generality of singular schemes. An example of how those properties can be used for very concrete computations can be found in the computation of Balmer and the first author of the Witt group of Grassmann varieties [4].

Let us now explain why we use triangulated monoidal methods, even though there is no mention of a tensor product in the definition of Witt groups of triangulated categories. In fact, the proof of many results amounts to verifying that a certain number of diagrams of morphisms of functors such as (2) below are commutative. It might be possible to check this by hand in every concrete situation; however, it would be extremely painful: try it for example in the simple case of a regular closed immersion. Hence, some kind of systematic method is needed. Our solution to this problem is the use of a convenient setting involving a tensor product, an adjoint internal Hom, functors of the type $f^{*}, f_{*}$ and $f^{!}$and the adjunction relationships between them, that is some variant of the so-called Grothendieck six functors formalism in an arbitrary triangulated category. In this setting, we have shown in [12] that all the necessary diagrams commute, whereas this article exploits the existence of this structure on 
various concrete triangulated categories. Here is a brief sketch of what is involved: Witt groups are defined for triangulated categories $\ell$ equipped with a duality, i.e. with a contravariant endofunctor $D$ on $\ell$ together with a bidual isomorphism of functors Id $\rightarrow D^{2}=D \circ D$ satisfying $D\left(\varpi_{A}\right) \circ \varpi_{D A}=\operatorname{Id}_{D A}$ for all objects $A$ of $\varphi$. A morphism between Witt groups is naturally induced by an exact functor $F: \ell_{1} \rightarrow \ell_{2}$ (both triangulated categories with dualities resp. $\left(D_{1}, \varpi_{1}\right)$ and $\left.\left(D_{2}, \varpi_{2}\right)\right)$ equipped with an isomorphism of exact functors $\phi: F D_{1} \rightarrow D_{2} F$ which explains how $F$ "commutes" with the dualities. The fact that the isomorphism $\phi$ is not the identity requires the analysis of its interactions with the other morphisms of functors involved. It is the central problem to solve when proving the main theorems. To start with, this morphism $\phi$ should make the diagram

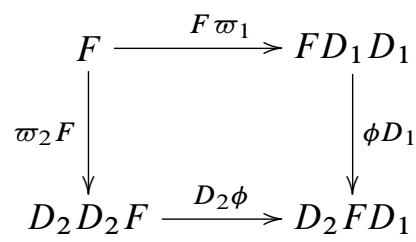

commutative. In [12], we discuss such morphisms of functors and diagrams in the setting of closed symmetric monoidal categories. More precisely, let $\ell_{1}$ and $\ell_{2}$ be closed symmetric monoidal categories, with tensor product denoted by $\otimes$ and internal Hom denoted by $[-,-]$. Given an object $K$, the functor $D_{K}:=[-, K]$ together with the canonical natural transformation $\varpi_{K}$ : Id $\rightarrow D_{K}^{2}$ defines a weak duality functor. Starting with an exact functor $f_{*}: \ell_{1} \rightarrow \ell_{2}$ which has a left adjoint $f^{*}$ (which is monoidal) and a right adjoint $f^{!}$, there is a natural transformation

$$
\zeta: f_{*} D_{f ! K} \rightarrow D_{K} f_{*}
$$

such that the diagram (1), which becomes

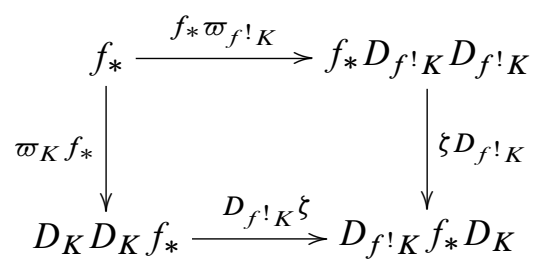

commutes, as shown in [12]. Therefore, provided $\varpi_{K}, \varpi_{f ! K}$ and $\zeta$ are isomorphisms, $f_{*}$ induces a morphism of Witt groups

$$
\mathrm{W}\left(e_{1}, D_{f ! K}, \varpi_{f ! K}\right) \rightarrow \mathrm{W}\left(e_{2}, D_{K}, \varpi_{K}\right)
$$

The present article is a description of how to apply this abstract closed monoidal setting to well-chosen derived categories of schemes, with the derived functors $\mathrm{L} f^{*}$, $\mathrm{R} f_{*}$ and its right adjoint $f^{!}$constructed by Grothendieck duality theory. 
The main result of this article is the definition of a push-forward along a proper morphism $f: X \rightarrow Y$ of separated noetherian schemes. In its most general form (Theorem 4.4), this push-forward is a morphism

$$
\mathrm{W}^{i}\left(X, f^{!} K\right) \stackrel{f_{*}}{\longrightarrow} \mathrm{W}^{i}(Y, K)
$$

where $K$ is a dualizing complex on $Y$. This push-forward is induced by the derived functor $\mathrm{R} f_{*}$ and a suitable morphism of functors $\zeta_{K}: \mathrm{R} f_{*} D_{f^{!} K} \rightarrow D_{K} \mathrm{R} f_{*}$. This means that a form on a complex $A$ for the duality $D_{f ! K}$ is sent to a form on the complex $\mathrm{R} f_{*} A$ for the duality $D_{K}$. We further prove that this push-forward respects composition (Theorem 5.3).

Similarly, for morphisms of finite tor-dimension $f$ we define a pull-back (Theorem 4.1), that is a morphism

$$
\mathrm{W}^{i}(Y, K) \rightarrow \mathrm{W}^{i}\left(X, \mathrm{~L} f^{*} K\right)
$$

respecting composition (Theorem 5.2).

We also prove a flat base change theorem (5.5) relating the push-forward and the pull-back in cartesian diagrams and a projection formula in the case of regular schemes (Theorem 5.7). Some explicit computations of transfers are provided in the last section.

We assume that schemes are separated and noetherian for the following technical reasons: quasi-compact and separated are necessary to have an equivalence between the derived category of quasi-coherent sheaves $D(\mathrm{Q} \operatorname{coh}(X))$ and the subcategory $D_{\mathrm{qc}}(X)$ of complexes with quasi-coherent homology in the derived category of all sheaves. Noetherian is used to ensure that the injectives in $\mathrm{Qcoh}(X)$ remain injective in the category of all $\mathcal{O}_{X}$-modules. Working without those assumptions would probably require significant improvements in the theory of Grothendieck duality; this is beyond the scope of this article which only intends to apply this theory to Witt groups.

Two main cases are discussed. The easier case is when all schemes considered are regular. Then their derived category $D_{\mathrm{b}, \mathrm{c}}$ of complexes with coherent and bounded homology is preserved under the derived tensor product $\otimes^{\mathrm{L}}$ and under RHom, the derived internal Hom. This endows $D_{\mathrm{b}, \mathrm{c}}$ with a natural structure of symmetric monoidal category. The dualizing complexes (see Definition 2.1) are line bundles or shifted line bundles. The coherent Witt groups are thus defined using the duality RHom $(-, L)$ for some line bundle $L$. Furthermore, the derived pull-back $\mathrm{L} f^{*}$ for any morphism, the derived push-forward $\mathrm{R} f_{*}$ and its right adjoint $f^{!}$for proper morphisms also preserve $D_{\mathrm{b}, \mathrm{c}}$. Hence the abstract formalism of [12] applies on the nose, and we therefore obtain push-forwards and their classical properties of composition, base change and projection.

The general case, when schemes are not assumed to be regular, is more complicated. Indeed, in this case $\otimes^{\mathrm{L}}$ or RHom do not necessarily preserve bounded 
homology and so there is no nice closed symmetric monoidal category structure on the category $D_{\mathrm{b}, \mathrm{c}}$ as the following affine example illustrates. Choose a field $k$ and set $X=\operatorname{Spec}\left(k[\epsilon] / \epsilon^{2}\right)$. Then consider the complex with $k$ concentrated in degree zero. A projective resolution of $k$ is given by

$$
\cdots \stackrel{. \epsilon}{\longrightarrow} k[\epsilon] / \epsilon^{2} \stackrel{. \epsilon}{\longrightarrow} k[\epsilon] / \epsilon^{2} \stackrel{. \epsilon}{\longrightarrow} k[\epsilon] / \epsilon^{2} \longrightarrow k \longrightarrow 0
$$

Thus $k \otimes^{\mathrm{L}} k$ is the complex

$$
\cdots \stackrel{0}{\longrightarrow} k \stackrel{0}{\longrightarrow} k \stackrel{0}{\longrightarrow} k \longrightarrow 0
$$

which has unbounded homology. On the other hand, the unbounded derived category $D_{\mathrm{qc}}$ of complexes with quasi-coherent cohomology admits a closed symmetric monoidal structure; this is not completely obvious, see Theorem 1.2. But this category is not suitable to define Witt groups, because there is no obvious (strong) duality on it and, anyway, as Eilenberg swindle type of arguments show for $K$-theory, unbounded categories are not the good framework to define cohomology theories. Still, the closed symmetric monoidal structure on $D_{\mathrm{qc}}$ is useful to prove systematically the commutativity of diagrams such as (1). That is, we can use the framework of [12] to prove this commutativity in the large closed symmetric monoidal category $D_{\mathrm{qc}}$ and then notice that all functors used in the definition of the duality $(\operatorname{RHom}(-, K)$ for some suitable $K)$ and the push-forward $\left(\mathrm{R} f_{*}\right)$ actually restrict to $D_{\mathrm{b}, \mathrm{c}}$ under mild additional assumptions. Thus, the commutativity of the diagrams involved is proved in large categories by general closed symmetric monoidal methods, but the diagrams actually often live in a smaller category whose Witt groups are interesting.

A technical point arising is the construction of the functors involved in the symmetric monoidal structure as well as $\mathrm{L} f^{*}, \mathrm{R} f_{*}$ and $f^{!}$on the unbounded derived category $D_{\mathrm{qc}}$. This relies on the work of Spaltenstein [35], the articles of Neeman [29] and [30], and on the very useful notes of Lipman [28], which are a reference on Grothendieck duality and contain very detailed explanations of all constructions.

The article is organized as follows. In Section 1, we recall the closed symmetric monoidal structures of the different categories we use. In Section 2, we use these structures to define triangulated categories with dualities and related Witt groups. Section 3 contains results on the derived functors $\mathrm{L} f^{*}$ and $\mathrm{R} f_{*}$ and on Grothendieck duality, i.e. the construction of the right adjoint $f !$ of $\mathrm{R} f_{*}$. Section 4 contains the main result of the paper, namely Theorem 4.4. We explain there explains how to use [12] to obtain the definition of push-forwards for the coherent Witt groups of schemes. It also contains a definition of the finite tor-dimension (e.g. flat) pull-back (Theorem 4.1). Section 5 explains the behavior of the push-forward and the pull-back under composition, and proves a base change formula relating them. Section 6 explains possible reformulations of the push-forward in different contexts and Section 7 studies in detail 
the push-forward in the case of finite field extensions, regular embeddings and projective bundles, which is useful for computations and also allows a comparison with the transfer maps of other authors when they are defined. Everything except some specific computations in the last section works both for Grothendieck-Witt groups $\mathrm{GW}$ and Witt groups W. For simplicity, we stated all results for W only.

The present article is a generalization of the main results of the unpublished preprints [10] and [11] on regular schemes. To keep this article short, some applications established in [11] (dévissage/localization, Witt motives and partial results about their decomposition for cellular varieties) are not included here. Most important, all the abstract theorems about triangulated symmetric monoidal functors and adjunctions between them which are crucial for proving the theorems of this article are proven in the long article [12].

We would like to thank Amnon Neeman for his precise explanations about his approach to dualizing complexes; it enabled us to generalize earlier versions of the results. We would also like to thank Paul Balmer and Bruno Kahn for their constant support, and the referee for his careful reading and detailed comments.

\section{Closed symmetric monoidal categories}

Let $S c h$ denote the category of separated noetherian schemes and $\mathcal{R} e g$ its full subcategory of regular schemes. For any scheme $X$, let $K(X)$ (resp. $D(X))$ denote the homotopy (resp. derived) category of homological complexes of $\mathcal{O}_{X}$-modules (without any restriction). We then add subscripts + for bounded above, i.e. bounded where the differentials go, - for bounded below, $b$ for bounded, i.e. below and above, $q c$ for quasi-coherent and $c$ for coherent to characterise the derived categories of complexes whose homology is as the subscript. For example $D_{\mathrm{b}, \mathrm{c}}(X)$ is the derived category of complexes of $\mathcal{O}_{X}$-modules with coherent and bounded homology, and $D_{\mathrm{qc}}(X)$ is the derived category of complexes with quasi-coherent homology. Note that we work with homological notation to be compatible with the literature on Witt groups, but it is easy to switch to cohomological notation by moving bounding subscripts to superscripts and exchanging + and - i.e. $D_{+}=D^{-}$.

For any scheme $X$, the usual tensor product $\otimes$ and internal Hom of complexes together with the obvious structure morphisms coming from the corresponding ones for sheaves turn $K(X)$ into a suspended closed symmetric monoidal category in the sense of Section 3 in [12]. This is completely classical and is detailed in [12], Appendix, where a discussion on sign choices can be found. In particular, we have a functor $T: K(X) \rightarrow K(X)$ given by $(T A)_{n}=T\left(A_{n-1}\right)$. 
Theorem 1.1. Let $X$ be a scheme.

(1) The tensor product on $K(X)$ admits a left derived functor

$$
\otimes^{\mathrm{L}}: D(X) \times D(X) \rightarrow D(X)
$$

together with unit, associativity and symmetry morphisms.

(2) It restricts to

$$
D_{\mathrm{qc}}(X) \times D_{\mathrm{qc}}(X) \rightarrow D_{\mathrm{qc}}(X) .
$$

(3) When $X \in \mathcal{R e g}$, it furthermore restricts to

$$
D_{\mathrm{b}, \mathrm{c}}(X) \times D_{\mathrm{b}, \mathrm{c}}(X) \rightarrow D_{\mathrm{b}, \mathrm{c}}(X) .
$$

(4) The internal Hom on $K(X)$ has a right derived functor $\mathrm{RHom}$

$$
D(X)^{o} \times D(X) \rightarrow D(X)
$$

which is a right adjoint to the derived tensor product in the usual special sense (natural in the three variables).

(5) When $X \in S c h$, RHom restricts to

$$
D_{\mathrm{b}, \mathrm{c}}(X)^{o} \times D_{\mathrm{b}, \mathrm{c}}(X) \rightarrow D_{\mathrm{c}}(X)
$$

as the usual RHom (computed by replacing the second variable by a quasiisomorphic complex of injectives in $\mathrm{Qcoh}(X)$ ).

(6) When $X \in \mathcal{R}$ eg, this last restricted RHom arrives in $D_{\mathrm{b}, \mathrm{c}}(X)$.

Proof. See Theorem A in [35] or [28], 2.5.7, for the existence of the derived tensor product. It is based on the existence of a q-flat (also called K-flat) resolution for any complex $C$, i.e. the existence of a quasi-isomorphism $Q_{C} \rightarrow C$ where $Q_{C}$ is a complex such that $\left(-\otimes Q_{C}\right)$ preserves quasi-isomorphisms. These resolutions can even be constructed functorially (see [28], 2.5.5). The derived tensor product can then be constructed by taking q-flat resolutions of both variables. The former case is used to define the unit morphism and the latter case to define the associativity and symmetry morphisms directly from the ones of $K(X)$ (see [35], Theorem A, or [28], 2.5.9). See [28], 2.5.8, for the fact that $\otimes^{\mathrm{L}}$ restricts to $D_{\mathrm{qc}}$. In the regular case, by Point (3) of Proposition A.4, we can replace any complex in $D_{\mathrm{b}, \mathrm{c}}(X)$ by a bounded complex of locally free sheaves, in which case the derived tensor product obviously maps to $D_{\mathrm{b}, \mathrm{c}}(X)$.

Similarly, the derived internal Hom is constructed using q-injective (also called Kinjective) resolutions: see Section 1 in [35] for the definition of a q-injective complex 
and [35], Theorem A, or [28], 2.4.5, for the existence of RHom. Adjointness is also stated in [35], Theorem A (see also [28], 2.6.1, for more details).

We now consider $\operatorname{RHom}(A, B)$ with $A, B \in D_{\mathrm{b}, \mathrm{c}}(X)$ for $X \in S c h$. By Corollary A.6, the right derived functor RHom here is computed as the one in [23], Proposition II.3.3. This proves Point (5). In the regular case, we can compute RHom by a locally free resolution of the first variable and then, up to isomorphism, also replace the second variable by a complex of locally free sheaves. As explained above, both these complexes can be chosen to be bounded, and since $\mathscr{H o m}(A, B)$ is coherent when $A$ and $B$ are [19], 5.3.5, this proves Point (6).

Now the subtle point is that $\operatorname{RHom}(M, N)$ is not necessarily an object in $D_{\mathrm{qc}}(X)$ when $M$ and $N$ are. To fix this, we use the quasicoherator $Q: \operatorname{Mod}(X) \rightarrow \operatorname{Qcoh}(X)$ as introduced in Lemma 3.2, p. 187 in [8], which is right adjoint to the inclusion Qcoh $(X) \subset \operatorname{Mod}(X)$. On an affine space $X=\operatorname{Spec}(A)$, it takes a sheaf of $\mathcal{O}_{X^{-}}$ modules to the quasi-coherent sheaf associated to the $A$-module of its global sections by the tilde construction. Its right derived functor is denoted by $\mathrm{R} Q$, as considered in [1], Remark 0.4, [28], Exercises 4.2.3, or B.16 in [36]. It is a right adjoint to the inclusion $D_{\mathrm{qc}}(X) \subseteq D(X)$, and $A \cong \mathrm{R} Q(A)$ when $A \in D_{-, \mathrm{qc}}(X)$ (in particular for $\left.A \in D_{\mathrm{b}, \mathrm{c}}(X)\right)$ by [8], Exposé II, Proposition 3.5.2. An alternative construction of $\mathrm{R} Q$ can be obtained from Theorem 4.1 in [29].

Theorem 1.2. Let $X$ be a scheme.

(1) The derived tensor product $\otimes^{\mathrm{L}}$ together with the obvious morphisms turns $D(X)$ into a symmetric monoidal category, closed by the RHom, and suspended in the sense of Section 3 in [12].

(2) If $X \in S c h$, the functor

$$
\mathrm{R} Q \circ \mathrm{RHom}: D_{\mathrm{qc}}(X)^{o} \times D_{\mathrm{qc}}(X) \rightarrow D_{\mathrm{qc}}(X)
$$

is a right adjoint (in the usual special way, see [26], (v), p. 97) to the restricted tensor product $\otimes^{\mathrm{L}}$ on $D_{\mathrm{qc}}$. This turns $D_{\mathrm{qc}}(X)$ into a suspended closed symmetric monoidal category.

(3) If $X \in \mathcal{R}$ eg, the usual RHom is a right adjoint (in the usual special way) to the restricted tensor product $\otimes^{\mathrm{L}}$ on $D_{\mathrm{b}, \mathrm{c}}$. This turns $D_{\mathrm{b}, \mathrm{c}}(X)$ into a suspended closed symmetric monoidal category.

Proof. The closed symmetric monoidal structure on $D(X)$ easily follows from Theorem 1.1. The fact that it is suspended follows, as explained in [12], Section 3, from the suspended bifunctor structure of RHom. The symmetric monoidal structure on $D_{\mathrm{qc}}(X)$ simply follows from the fact that $\otimes^{\mathrm{L}}$ restricts to it. The fact that it is closed 
is a formal consequence of the fact that $D(X)$ is closed and that $\mathrm{R} Q$ is a right adjoint to the (monoidal) inclusion $\iota: D_{\mathrm{qc}}(X) \subseteq D(X)$ :

$$
\begin{gathered}
\operatorname{Hom}_{\mathrm{qc}}\left(A \otimes^{\mathrm{L}} B, C\right)=\operatorname{Hom}\left(\iota\left(A \otimes^{\mathrm{L}} B\right), \iota C\right) \simeq \operatorname{Hom}\left(\iota A \otimes^{\mathrm{L}} \iota B, \iota C\right) \\
\simeq \operatorname{Hom}(\iota A, \operatorname{RHom}(\iota B, \iota C)) \simeq \operatorname{Hom}_{\mathrm{qc}}(A, \operatorname{R} Q \operatorname{RHom}(\iota A, \iota B)) .
\end{gathered}
$$

(The closedness - that is the existence of the right adjoint to the derived tensor product - can also be deduced from Brown representability, in the spirit of the examples following [29], Theorem 4.1.) Point (3) follows from the same considerations, using Theorem 1.1 (3) and (6).

Notation 1.3. To shorten the notation, let $[-,-]$ denote the functor RHom, right adjoint to the tensor product on the derived category $D$ and let $[-,-]^{\prime}$ denote the functor $\mathrm{R} Q \circ \mathrm{RHom}$, right adjoint to the tensor product on the derived category $D_{\mathrm{qc}}$.

Since the derived quasi-coherator is the identity on $D_{-, \mathrm{qc}}$ (see above), if $[A, B] \in$ $D_{-, \mathrm{qc}}$ then $[A, B] \cong[A, B]^{\prime}$.

We finish this section by pointing out a comment of Neeman: exploiting the fact that for $X \in S c h$, there are enough flat objects in $D_{\mathrm{qc}}$ and his representability result more extensively gives an alternative approach for constructing a closed symmetric monoidal structure on $D_{\mathrm{qc}}(X)$ directly without passing through $D(X)$.

\section{Witt groups}

From now on, we assume that all schemes are defined over $\mathbb{Z}[1 / 2]$.

To define a Witt group, we need a strong duality on a triangulated category. Using the previous framework of triangulated closed symmetric monoidal categories, we recall how $[-, K]$ and $[-, K]^{\prime}$ define dualities. The purpose of this section is to compare the restrictions of these dualities to the subcategory $D_{\mathrm{b}, \mathrm{c}}$ and to discuss when these dualities are strong on it. For any object $K$, let $\sharp_{K}$ (resp. $\sharp_{K}^{\prime}$ ) denote the contravariant exact functor $[-, K]$ (resp. $[-, K]^{\prime}$ ).

Following [12], Section 3.2, applied to the closed symmetric monoidal structure on $D(X)$ with $X$ an arbitrary scheme, we may define the bidual morphism

$$
\varpi_{K}: \mathrm{Id} \rightarrow \sharp_{K} \sharp_{K}
$$

as a morphism of triangulated endofunctors of $D(X)$. From Corollary 3.2 in [12], we obtain that $\left(D(X), \sharp_{K}, \varpi_{K}\right)$ is a triangulated category with weak duality (in the sense of [12], Definition 2.1.1, so $\varpi_{K}$ is not necessarily an isomorphism). Similarly, when $X \in S c h$, we obtain a triangulated category with weak duality $\left(D_{\mathrm{qc}}(X), \sharp_{K}^{\prime}, \varpi_{K}^{\prime}\right)$. 
Definition 2.1. Let $K$ be an object of $D_{\mathrm{qc}}(X)$. It is a dualizing complex (or it is dualizing) if

- the functor $[-, K]$ preserves $D_{\mathrm{b}, \mathrm{c}}(X)$ and

- the bidual morphism $\varpi_{K}$ is an isomorphism on $D_{\mathrm{b}, \mathrm{c}}(X)$.

If furthermore it has finite injective dimension, i.e. it is quasi-isomorphic to a finite complex of injectives, we say it is an injectively bounded dualizing complex.

In the terminology of Definition 2.1.1 in [12], the second condition says that $\sharp_{K}$ is a strong duality on the subcategory $D_{\mathrm{b}, \mathrm{c}}(X)$.

Note that for any $X \in S c h$, a dualizing complex $K$ is automatically in $D_{\mathrm{b}, \mathrm{c}}(X)$ since the natural morphism $K \rightarrow\left[\mathcal{O}_{X}, K\right]$ coming from the monoidal structure is an isomorphism and $\mathcal{O}_{X}$ is coherent. In particular, our definition is exactly the "modern" (Definition 3.1, [30]) by Lemma 3.5 of loc. cit. Also note that our injectively bounded dualizing complexes are the "old" dualizing complexes of [23], V. §2.

Proposition 2.2. Let $X \in S c h$ and $K \in D_{\mathrm{qc}}(X)$ be a dualizing complex. Then the functors $\sharp_{K}$ and $\sharp_{K}^{\prime}$ coincide and the bidual morphisms $\varpi_{K}$ and $\varpi_{K}^{\prime}$ are equal on the subcategory $D_{\mathrm{b}, \mathrm{c}}(X)$.

Proof. Since $[A, K] \in D_{\mathrm{b}, \mathrm{c}}(X)$ for any $A \in D_{\mathrm{b}, \mathrm{c}}(X)$, we have $[A, K]^{\prime} \cong[A, K]$ by the remark after Notation 1.3 which proves that $\sharp_{K}^{\prime} \cong \sharp_{K}$. The bidual morphisms are then equal by the large commutative diagram considered in the proof of Theorem 4.1.2 in [12], in which the $f^{*}$ should be replaced by the inclusion $D_{\mathrm{qc}}(X) \subset D(X)$, which is monoidal by definition of the tensor product on $D_{\mathrm{qc}}(X)$.

Example 2.3. (1) A dualizing complex tensored by a shifted line bundle is still a dualizing complex. In fact, this is the only freedom: by Lemma 3.9 in [30] (see also [23], Theorem V.3.1, for the injectively bounded case), a dualizing complex is unique up to tensoring by shifted line bundles (the shift can be different on different connected component of $X$ ).

(2) On a Gorenstein scheme $X$ (e.g. regular), $\mathcal{O}_{X}$ itself is dualizing, so by the previous point, the only dualizing complexes are the shifted line bundles.

Note that on a regular scheme, the category $D_{\mathrm{b}, \mathrm{c}}(X)$ itself is closed symmetric monoidal. It follows that dualizing complexes are dualizing objects in the sense of Definition 3.2.2 in [12] in the category $D_{\mathrm{b}, \mathrm{c}}(X)$, for $X \in S c h$.

Theorem 2.4. Let $X \in$ Sch and $K$ be dualizing. Then $\left(D_{\mathrm{b}, \mathrm{c}}(X), \sharp_{K}, \varpi_{K}\right)$ is a triangulated category with strong duality in the sense of Definition 2.1.1 in [12]. Let it be denoted by $\mathcal{C}_{K}$ and its Witt groups by $\mathrm{W}^{i}(X, K), i \in \mathbb{Z}$ ([12], Definition 2.1.5). 
Proof. The functor $\sharp_{K}=\sharp_{K}^{\prime}$ is a contravariant endofunctor of $D_{\mathrm{b}, \mathrm{c}}(X)$ and $\varpi_{K}=$ $\varpi_{K}^{\prime}$ is an isomorphism on this category by definition of dualizing complexes by Proposition 2.2. The necessary commutative diagrams that $\sharp_{K}^{\prime}$ and $\varpi_{K}^{\prime}$ must satisfy simply follow from the fact that they are already satisfied in $D_{\mathrm{qc}}(X)$ since $\left(D_{\mathrm{qc}}(X), \sharp_{K}^{\prime}, \varpi_{K}^{\prime}\right)$ is a triangulated category with weak duality.

We may thus think of the triangulated category with duality $\left(D_{\mathrm{b}, \mathrm{c}}(X), \sharp_{K}, \varpi_{K}\right)$ as being restricted from $\left(D(X), \sharp_{K}, \varpi_{K}\right)$ or from $\left(D_{\mathrm{qc}}(X), \sharp_{K}^{\prime}, \varpi_{K}^{\prime}\right)$, both structures coinciding on $D_{\mathrm{b}, \mathrm{c}}(X)$.

Remark 2.5. In [2], all dualities considered are strict, i.e. they strictly commute with the suspension, but this assumption is only there for simplicity. Instead, in Definition 2.1.1 of [12], we only assume commutativity up to a natural isomorphism, and all theorems in [2] are still true in this more general situation.

Remark 2.6. Recall (see e.g. [40], Definition 10.5.1) that for a left exact functor $f$ between exact categories, the right derived functor really is a couple $(\mathrm{R} f, s)$ with $s: q f \rightarrow(\mathrm{R} f) q$ and $q$ the morphism from the homotopy category to the derived category. It is only the couple $(\mathrm{R} f, s)$ which is unique up to unique isomorphism and therefore deserves being called the right derived functor, despite the standard abbreviated notation $\mathrm{R} f$. Consequently, the various derived functors, for example $\mathrm{RHom}(-, K)$ (used to define the duality) and $\mathrm{R} f_{*}$ (used below to define the pushforward) together with all the morphisms of functors defining the symmetric monoidal structure can be considered as abstract exact functors and morphisms of exact functors. With them, it is possible to define coherent Witt groups and push-forwards by the methods discussed in this article, since these methods only involve the abstract triangulated categories and functors, i.e. the framework of [12]. But as such, there is no uniqueness of all these constructions. It is only if we keep as extra data all the structural morphisms of the derived functors (the $s$ part of the couples), and thus the relationship between the closed symmetric monoidal structure on $K(X)$ and the one on $D(X)$, that the whole derived construction becomes unique up to unique isomorphism, thus in particular the induced dualities, pull-backs and push-forwards.

\section{The functors $\mathrm{L} f^{*}, \mathrm{R} f_{*}$ and $f^{!}$}

We now introduce the functors $\mathrm{L} f^{*}, \mathrm{R} f_{*}$ and $f^{!}$associated to a morphism of schemes $f$ and explain how they behave with respect to the monoidal structures. The first two functors are derived functors, whereas the third one is right adjoint to $\mathrm{R} f_{*}$ at the level of derived categories, but is not the derived functor of some underlying functor on the category of $\mathcal{O}_{X}$-modules. The construction of $f^{!}$is the heart of Grothendieck duality theory, for which we refer the reader to [23], [37], [29], [13] or [28]. 
Proposition 3.1. Let $f: X \rightarrow Y$ be a morphism of schemes.

(1) The functor $f^{*}$ admits a left derived functor $\mathrm{L} f^{*}: D(Y) \rightarrow D(X)$ which restricts to $D_{\mathrm{qc}}(Y) \rightarrow D_{\mathrm{qc}}(X)$.

(2) If $f$ is of finite tor-dimension (see e.g. Examples (2.7.6), [28]) or if $X, Y \in \mathcal{R e g}$, then $\mathrm{L} f^{*}$ restricts to $D_{\mathrm{b}, \mathrm{c}}(Y) \rightarrow D_{\mathrm{b}, \mathrm{c}}(X)$.

Proof. For existence, see [35], Theorem A (iii) or Proposition 6.7, or Example 2.7.3 in [28]. For the fact that it restricts to $D_{\mathrm{qc}}$, see [28], 3.9.1. It restricts to $D_{\mathrm{b}, \mathrm{c}}$ in the finite tor-dimension case because $\mathrm{L} f^{*}$ is then bounded and it respects the coherence of the cohomology by [23], Proposition II.4.4, bearing in mind Proposition A.7. The case $X, Y \in \mathcal{R e g}$ follows from Point (3) of Proposition A.4 and Proposition A.7.

Proposition 3.2. The usual isomorphism $f^{*}(A \otimes B) \rightarrow f^{*} A \otimes f^{*} B$ induces an isomorphism of triangulated bifunctors (in the sense of [12], Definition 1.4.14)

$$
\alpha: \mathrm{L} f^{*}(-\otimes-) \rightarrow \mathrm{L} f^{*}(-) \otimes \mathrm{L} f^{*}(-)
$$

which turns $\mathrm{L} f^{*}$ into a suspended symmetric monoidal functor in the sense of [12], Section 4.

Proof. See [35], Proposition 6.8. The morphism $\alpha$ is defined as the corresponding one on $K(X)$ after having replaced both variables by q-flat resolutions. It is already an isomorphism on $K(X)$. The commutative diagrams required (compatibility with the associativity, unit and symmetry of the monoidal structures) then easily follow from the corresponding ones on $K(X)$, using Proposition A.3, Points (1) and (3).

By Proposition 4.1.1 in [12] applied to the symmetric monoidal structure and $\mathrm{L} f^{*}$ on $D(X)$, there is a natural morphism

$$
\beta: \mathrm{L} f^{*}[-,-] \rightarrow\left[\mathrm{L} f^{*}(-), \mathrm{L} f^{*}(-)\right] .
$$

We also obtain a morphism

$$
\beta^{\prime}: \mathrm{L} f^{*}[-,-]^{\prime} \rightarrow\left[\mathrm{L} f^{*}(-), \mathrm{L} f^{*}(-)\right]^{\prime} .
$$

using $D_{\mathrm{qc}}(X)$ instead of $D(X)$.

Proposition 3.3. Let $X, Y \in$ Sch and $A, B \in D_{\mathrm{qc}}(Y)$. Assuming $[A, B] \in D_{\mathrm{qc}}(Y)$ and $\left[\mathrm{L} f^{*} A, \mathrm{~L} f^{*} B\right] \in D_{\mathrm{qc}}(X)$, the morphisms $\beta_{A, B}$ and $\beta_{A, B}^{\prime}$ coincide. In particular, when $K$ and $\mathrm{L} f^{*} K$ are dualizing, $\beta_{K}$ and $\beta_{K}^{\prime}$ coincide. 
Proof. This follows from the commutative diagram

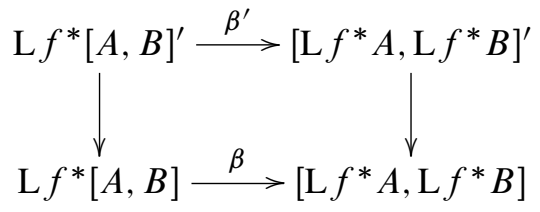

in which the vertical maps become identities under the assumptions. This diagram is formally obtained from the definitions of $\beta$ and $\beta^{\prime}$ out of the closed monoidal structures.

Proposition 3.4. When $X, Y \in$ Sch and $f: X \rightarrow Y$ is of finite tor-dimension or when $X, Y \in \operatorname{Reg}$ and for any $f: X \rightarrow Y$, the natural morphism $\beta$ is an isomorphism on objects in $D_{\mathrm{b}, \mathrm{c}}$.

Proof. This follows from [28], Proposition 4.6.6, for $f$ of finite tor-dimension, the first variable coherent and the second in $D_{-}$, so in particular for both in $D_{\mathrm{b}, \mathrm{c}}$. Note that the $\rho$ of loc. cit. coincides with our $\beta$ by definition (compare [28], (3.5.4.5), and [12], Proposition 4.1.1). In the regular case, by Point (3) of Proposition A.4, we can assume our objects are bounded complexes of locally free sheaves, in which case the result follows from [28], Proposition 4.6.7.

Proposition 3.5. Let $f: X \rightarrow Y$ be a morphism of schemes.

(1) The functor $f_{*}$ admits a right derived functor $\mathrm{R} f_{*}: D(X) \rightarrow D(Y)$.

(2) The functor $\mathrm{R} f_{*}$ restricts to $D_{\mathrm{qc}}(X) \rightarrow D_{\mathrm{qc}}(Y)$ when $f$ is quasi-compact and separated, in particular if $X$ and $Y$ are in Sch, see Corollary 6.1.10 in [22].

(3) The functor $\mathrm{R} f_{*}$ restricts to $D_{\mathrm{b}, \mathrm{c}}(X) \rightarrow D_{\mathrm{b}, \mathrm{c}}(Y)$ when $f$ is proper and $Y$ is quasi-compact.

Proof. For existence, see Theorem A (iii) in [35] or Examples 2.7.3 in [28]. For the fact that it restricts to $D_{\mathrm{qc}}(-)$ see [28], 3.9.2. In the proper case with $Y$ quasicompact, it restricts to $D_{\mathrm{b}, \mathrm{qc}}(-)$ by [28],3.9.2, and it then preserves coherence of the cohomology by Theorem 3.2.1 in [21]. Note that we use Definition 5.3.1 in [19] to define coherent modules on non necessarily noetherian schemes.

Proposition 3.6. For any morphism $f$ of schemes, the functor $\mathrm{R} f_{*}$ is a right adjoint to $\mathrm{L} f^{*}$ on $D(-)$ and consequently on all full subcategories to which both functors restrict.

Proof. See see Theorem A (iii) in [35] or Proposition 3.2.1 in [28]. 
By Proposition 4.2.5, [12] applied to the monoidal structure and the functors on the categories $D(-)$, we obtain the projection morphism

$$
\pi: \mathrm{R} f_{*}(-) \otimes-\rightarrow \mathrm{R} f_{*}\left(-\otimes \mathrm{L} f^{*}(-)\right)
$$

Theorem 3.7. Let $f: X \rightarrow Y$ be quasi-compact and quasi-separated e.g. proper. Then the projection morphism $\pi$ is an isomorphism on $D_{\mathrm{qc}}$.

Proof. This is Proposition 3.9.4 in [28].

Theorem 3.8. For any separated morphism $f: X \rightarrow Y$ with $X$ and $Y$ separated and quasi-compact, the functor $\mathrm{R} f_{*}: D_{\mathrm{qc}}(X) \rightarrow D_{\mathrm{qc}}(Y)$ has a right adjoint $f !$.

Proof. See Example 4.2 in [29] and use that $D_{\mathrm{qc}}(-)$ and $D(\mathrm{Q} \operatorname{coh}(-))$ are equivalent for separated quasi-compact schemes by Proposition A.4 (1).

Proposition 3.9. Let $f: X \rightarrow Y$ be a proper morphism of separated noetherian schemes and let $K$ be a dualizing complex on $Y$. Then $f^{!} K$ is a dualizing complex on $X$. If $K$ is an injectively bounded dualizing complex i.e. dualizing in the sense of [23], V. $\S 2$, then $f^{!} K$ is injectively bounded too.

Proof. For the case of injectively bounded complexes, see [23], V, §8, or Corollary 3, [37]. For the general case, we reproduce here a proof of Neeman. Since the question of whether $f^{!} K$ is dualizing is local on $X$, we may assume $Y$ is affine and restrict to an affine open set $U$ of $X$. As $f$ is of finite type, we have a factorization $U \rightarrow$ $\mathbb{A}^{n} \times Y \rightarrow Y$ for some $n$ where the left arrow is a closed embedding. Taking the closure of $U$ in $\mathbb{P}^{n} \times Y$, we see that $U$ can be embedded as an open subset of a closed subset of some $Y \times \mathbb{P}^{n}$. Hence we only have to show that closed immersions, open immersions and projections $Y \times \mathbb{P}^{n} \rightarrow Y$ respect dualizing complexes. The case of closed immersions is done in [30], see Theorem 3.14, Remark 3.17 and Lemma 3.18; closed immersions are finite. The case of open immersions is Theorem 3.12 in [30]. For projective morphisms $f: \mathbb{P}_{Y}^{n} \rightarrow Y$, one uses that $\operatorname{RHom}\left(A, f^{!} K\right) \cong$ $\operatorname{RHom}\left(A, f^{!} \mathcal{O} \otimes f^{*} K\right) \cong \operatorname{RHom}\left(A, f^{*} K\right) \otimes f^{!} \mathcal{O}$, using Lemma 5.6 below and that $f^{!} \mathcal{O}$ is a shifted line bundle by [23], Section VII.4. Then one checks the conditions of Definition 2.1 on objects of the form $f^{*} B$ and $\mathcal{O}(i)$ which by a theorem of Beilinson [7] generate $D_{\mathrm{b}, \mathrm{c}}\left(\mathbb{P}_{Y}^{n}\right)$ as a thick triangulated category.

\section{Pull-back and push-forward for Witt groups}

We can now state the main result of this article: the definition of the push-forward for coherent Witt groups along proper morphisms (Theorem 4.4). This section also 
contains a definition of the pull-backs for morphisms of finite tor-dimension (Theorem 4.1).

Let $f: X \rightarrow Y$ be a morphism of schemes. By [12], Theorem 4.1.2, applied to the monoidal categories $D(-), \beta_{K}: \mathrm{L} f^{*} \sharp_{K} \rightarrow \sharp_{\mathrm{L} f}{ }^{*} K_{K} \mathrm{~L} f^{*}$ defines a duality preserving functor $\left\{\mathrm{L} f^{*}, \beta_{K}\right\}$ between triangulated categories with weak dualities, from $\left(D(Y), \sharp_{K}, \varpi_{K}\right)$ to $\left(D(X), \sharp_{\mathrm{L} f * K}, \varpi_{\mathrm{L} f *_{K}}\right)$, for any object $K$ of $D_{\mathrm{qc}}(Y)$.

Theorem 4.1. Let $f: X \rightarrow Y$ be a morphism of schemes such that

- the objects $K$ and $\mathrm{L} f^{*} K$ are dualizing.

- $\mathrm{L} f^{*}$ preserves $D_{\mathrm{b}, \mathrm{c}}$,

- $\beta_{K}$ is an isomorphism on $D_{\mathrm{b}, \mathrm{c}}(Y)$,

Then $\left\{\mathrm{L} f^{*}, \beta_{K}\right\}$ induces a morphism on Witt groups,

$$
f^{*}: \mathrm{W}^{i}(Y, K) \rightarrow \mathrm{W}^{i}\left(X, \mathrm{~L} f^{*} K\right),
$$

which we call pull-back. This pull-back therefore exists in particular if $K$ and $\mathrm{L} f^{*} K$ are dualizing and

- $f$ is of finite tor-dimension and $X, Y \in S c h$ or

- for any $f$ and $X, Y \in \mathcal{R e g}$ in which case $K$ dualizing implies $\mathrm{L} f^{*} K$ dualizing by Example 2.3.

Proof. This follows from Theorem 4.1.2 and Lemma 2.2.6(1) in [12]. The theorem of loc. cit. ensures the existence of the appropriate commutative diagrams in $D(X)$. The requirements in the lemma of loc. cit. that the dualities given by $K$ and $f^{*} K$ restrict as strong dualities to $D_{\mathrm{b}, \mathrm{c}}$ are satisfied by assumption, and the requirement that $\beta_{K}$ is an isomorphism when restricted to $D_{\mathrm{b}, \mathrm{c}}$ follows from Proposition 3.4.

Remark 4.2. Note that we obtain the very same pull-back when starting with the monoidal structure on $D_{\mathrm{qc}}$ instead of $D$. This follows from Proposition 3.3.

Remark 4.3. In Theorem 3.12 of [30] it is proved that if $f$ is an open immersion, then $\mathrm{L} f^{*} K$ is automatically dualizing if $K$ is dualizing.

Let $X, Y \in S c h$, let $K \in D_{\mathrm{qc}}(Y)$ and let $f: X \rightarrow Y$ be a separated morphism. From [12], Theorem 4.2.9, applied to the closed monoidal category $D_{\mathrm{qc}}(X)$, we obtain a morphism of functors $\zeta_{K}: \mathrm{R} f_{*} \sharp_{f ! K}^{\prime} \rightarrow \sharp_{K}^{\prime} \mathrm{R} f_{*}$. By loc. cit., the pair $\left\{\mathrm{R} f_{*}, \zeta_{K}\right\}$ is duality preserving, i.e. Diagram (1) commutes. 
Theorem 4.4. Let $X, Y \in$ Sch and $f: X \rightarrow Y$ be a separated morphism such that $\mathrm{R} f_{*}$ preserves $D_{\mathrm{b}, \mathrm{c}}$. Let $K$ and $f^{!} K$ be dualizing. Then $\left\{\mathrm{R} f_{*}, \zeta_{K}\right\}$ induces a morphisms of Witt groups

$$
f_{*}: \mathrm{W}^{i}\left(X, f^{!} K\right) \rightarrow \mathrm{W}^{i}(Y, K)
$$

which we call push-forward. This push-forward is therefore defined in particular if $f$ is proper and $K$ is dualizing (see Proposition 3.9).

Proof. This follows from Theorem 4.2.9 and Lemma 2.2.6.(1) in [12]. For the theorem of loc. cit., consider the triangulated closed monoidal category $D_{\mathrm{qc}}$. The fact that $\zeta_{K}$ is an isomorphism follows from Proposition 4.3.3 in [12] using Theorem 3.7. Then, apply the Lemma of loc. cit. to the subcategories $D_{\mathrm{b}, \mathrm{c}}$, to which the dualities restrict by definition of a dualizing object. Note that when $X$ and $Y$ are regular, the complete proof works using directly $D_{\mathrm{b}, \mathrm{c}}$ as the triangulated closed monoidal category in Theorem 4.2 .9 of [12].

\section{Properties}

We now show that both push-forwards and pull-backs respect composition and that they commute in an appropriate way ("base change") provided certain standard conditions hold. We also prove a projection formula for regular schemes.

Theorem 5.1. For any $f: X \rightarrow Y$ and $g: Y \rightarrow Z$,

(1) there is an isomorphism $\mathrm{L} f^{*} \circ \mathrm{L} g^{*} \rightarrow \mathrm{L}(g \circ f)^{*}$ between functors on $D(-)$ which is associative in the usual sense.

(2) There is an isomorphism $\mathrm{R}(g \circ f)_{*} \rightarrow \mathrm{R} g_{*} \circ \mathrm{R} f_{*}$ between functors on $D(-)$ which is associative in the usual sense, and respects the adjoint couple $\left(\mathrm{L}(-)^{*}, \mathrm{R}(-)_{*}\right)$ in the sense of Definition 5.1 .5 of $[12]$.

(3) When the schemes are separated and quasi-compact, and both $f$ and $g$ are separated, there is an isomorphism $f^{!} \circ g^{!} \rightarrow(g \circ f)^{!}$between functors on $D_{\mathrm{qc}}(-)$ which is associative in the usual sense, and which respects the adjoint couple $\left(\mathrm{R}(-)_{*},(-)^{!}\right)$in the sense of Definition 5.1.5 of [12].

Proof. For the functors $\mathrm{L} f^{*}$ on $D$, the isomorphism is in Theorem A (iii), [35] or [28], 3.6.4. For a proof that it is associative, see Scholium 3.6.10 in [28]. The other points follow from the first one by Lemma 5.1.6 in [12]. 
Theorem 5.2. The pull-back respects composition: the diagram

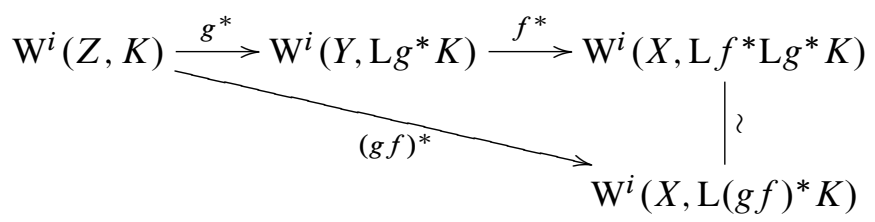

commutes, under the conditions for the existence of the pull-backs $f^{*}$ and $g^{*}$ of Theorem 4.1.

Proof. This follows from [12], Theorem 5.1.3 and Corollary 5.1.4, applied to the structures on $D(-)$.

Theorem 5.3. The push-forward respects composition: the diagram

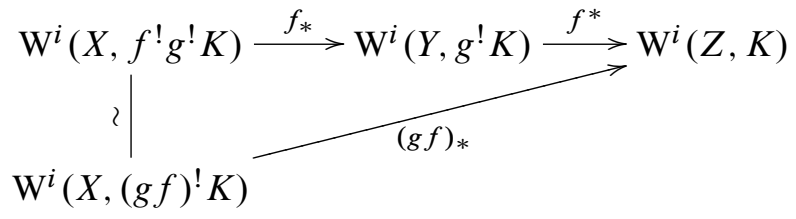

commutes, under the conditions for the existence of the push-forward of Theorem 4.4.

Proof. This follows from [12], Theorem 5.1.9 and Corollary 5.1.10, applied to the structures on $D_{\mathrm{qc}}(-)$.

We now prove a base change formula. Let us consider a pull-back diagram

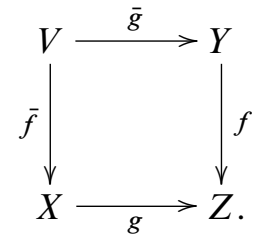

By Section 5.2, [12], we obtain a morphism of functors

$$
\varepsilon: \mathrm{L}^{*} \mathrm{R} g_{*} \rightarrow \mathrm{R} \bar{g}_{*} \mathrm{~L} \bar{f}^{*}
$$

between functors on $D(X)$.

Proposition 5.4. If all schemes are in Sch and the diagram is tor-independent, e.g. $f$ flat, the morphism $\varepsilon$ is an isomorphism on $D_{\mathrm{qc}}(X)$. 
Proof. The case where $f$ is flat is Proposition 3.9.5 in [28] (all maps between schemes in $\delta$ ch are "concentrated" in the sense of loc. cit.). The more general case is Theorem 3.10 .3 in [28].

Then, when the schemes are in $\delta c h$, still by Section 5.2 of [12], $\varepsilon$ induces a morphism

$$
\gamma: \mathrm{L} \bar{f}^{*} g^{!} \rightarrow \bar{g}^{!} \mathrm{L} f^{*}
$$

between functors on $D_{\mathrm{qc}}(X)$. It is an isomorphism on the subcategory $D_{-, \mathrm{qc}}$ by Corollary 4.4.3, [28]. In particular, $\gamma_{K}$ is an isomorphism when $K$ is dualizing (and thus in $\left.D_{\mathrm{b}, \mathrm{c}}(Z)\right)$.

Theorem 5.5 (Base change). Under the assumptions of Proposition 5.4 and the ones for the pull-backs along $f$ and $\bar{f}$ and the push-forwards along $g$ and $\bar{g}$ to exist (Theorems 4.1 and 4.4), the pull-back and push-forward satisfy a base change formula: the diagram

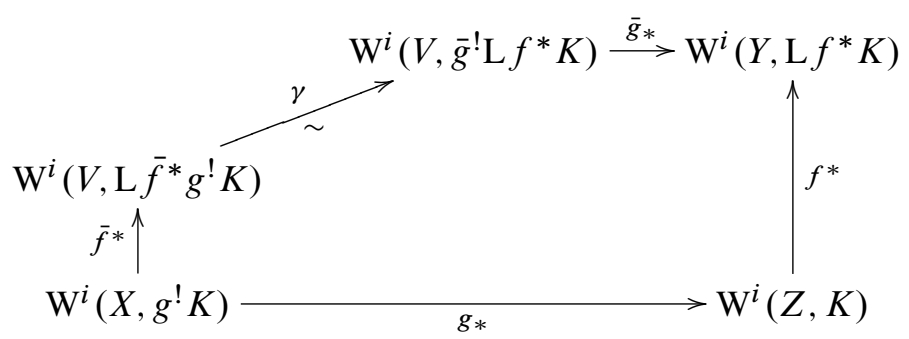

commutes.

Proof. This follows from [12], Theorem 5.2.1 and Corollary 5.2.2, applied to the structures on $D_{\mathrm{qc}}(-)$, keeping in mind Remark 4.2.

We conclude this section with a projection formula for Witt groups, in the case of regular schemes. For this, we first need to introduce another natural morphism that will anyway be of some use even in the case of non regular schemes.

When $f: X \rightarrow Y$ is a separated morphism in $\delta c h$, using the functors $\mathrm{L} f^{*}$ (monoidal), $\mathrm{R} f_{*}$ and $f^{!}$between the categories $D_{\mathrm{qc}}(X)$ and $D_{\mathrm{qc}}(Y)$, and the fact that the projection morphism $\pi$ is an isomorphism by Theorem 3.7, we obtain a morphism of functors

$$
\theta: f^{!}(-) \otimes^{\mathrm{L}} \mathrm{L} f^{*}(-) \rightarrow f^{!}\left(-\otimes^{\mathrm{L}}-\right)
$$

by Proposition 4.3.1 in [12].

Lemma 5.6. The morphism $\theta: f^{!} A \otimes^{\mathrm{L}} \mathrm{L} f^{*} B \rightarrow f^{!}\left(A \otimes{ }^{\mathrm{L}} B\right)$ is an isomorphism when $B$ is a perfect complex. 
Proof. The morphism $\theta$ is compatible with open immersions by Diagram 41 of Proposition 5.2.5 in [12], and so we can restrict to the case of bounded complexes of vector bundles, then to vector bundles, then again using open immersions to the trivial bundle $\mathcal{O}_{Y}$. In that case, one can show that $\theta_{A, \mathcal{O}_{Y}}$ coincides with the unit morphism of the monoidal structure $f^{!}(A) \otimes^{\mathrm{L}} \mathcal{O}_{X} \rightarrow f^{!}(A)$, and is therefore an isomorphism. By this coincidence we mean that the left diagram
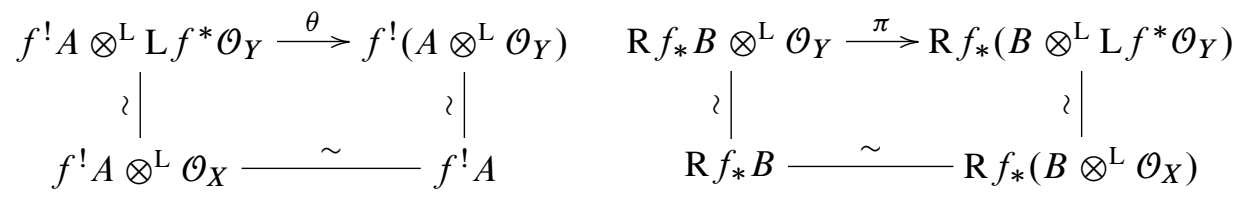

is commutative, in which the left vertical morphism is the fact that $\mathrm{L} f^{*}$ is monoidal and in particular respects units, and the bottom and right maps are unit morphisms of the monoidal structures. By following the definition of $\theta$ given in Proposition 4.3.1 of [12] the commutativity of the left diagram follows from the one of the right hand side, which is in turn implied, using the definition of $\pi$ in Proposition 4.2.5 of [12], by the compatibility of the unit and monoidal structure morphisms for $\mathrm{L} f^{*}$.

For any scheme $X$ in $\mathcal{R e g}$, the derived tensor product preserves $D_{\mathrm{b}, \mathrm{c}}(X)$ (Theorem 1.1(3)). This gives two different products on Witt groups by the formalism of [18], using Proposition 4.4.6 and Corollary 4.4.7 in [12] applied to the closed monoidal structure of $D_{\mathrm{b}, \mathrm{c}}(X)$. We fix one of these products (say, the left one) for the following results. When $K$ and $L$ are shifted line bundles, thus dualizing complexes, the product is a pairing

$$
\mathrm{W}^{i}(X, K) \times \mathrm{W}^{j}(X, L) \rightarrow \mathrm{W}^{i+j}(X, K \otimes L) .
$$

Theorem 5.7 (Projection formula). For any proper morphism $f: X \rightarrow Y$ with $X, Y \in \mathcal{R e g}$, the pull-back and push-forward satisfy a projection formula: If $K$, $L$ are shifted line bundles on $Y, x \in \mathrm{W}^{i}\left(X, f^{!} K\right)$ and $y \in \mathrm{W}^{j}(Y, L)$, then $f_{*}\left(I\left(x . f^{*} y\right)\right)=f_{*}(x) \cdot y$ in $\mathrm{W}^{i+j}(Y, K \otimes L)$ with $I$ the isomorphism from $\mathrm{W}^{i}\left(X, f^{!} K \otimes f^{*} L\right)$ to $\mathrm{W}^{i}\left(X, f^{!}(K \otimes L)\right)$ induced by $\theta_{K, L}$.

Proof. First note that $L$ being a shifted line bundle explains the absence of derivations in the pull-back and tensor products above. Then, the morphism $\theta_{K, L}$ is an isomorphism by Lemma 5.6, thus the result follows from [12], Theorem 5.5.1 and Corollary 5.5.2, applied to the closed monoidal structure on $D_{\mathrm{b}, \mathrm{c}}$.

\section{Reformulations in special cases}

In this section, we give other canonical ways of writing the push-forward, under additional assumptions. 
Notation 6.1. For an equidimensional morphism $f: X \rightarrow Y$ of relative dimension $n$, let $\omega_{f}$ denote the object $f^{!}\left(\mathcal{O}_{Y}\right)[-n]:=T^{-n} f^{!}\left(\mathcal{O}_{Y}\right)$.

This notation is motivated by the fact that in several cases, this object can be identified with a geometric object called a relative dualizing sheaf and usually denoted $\omega_{f}$ : see Sections 7.2 and 7.3 for the examples of regular embeddings and projective spaces.

\subsection{Relative dualizing sheaf}

Theorem 6.2. Let $f: X \rightarrow Y$ be a proper morphism in $S c h, K$ a dualizing complex on $Y$ such that $f ! K$ is a dualizing complex and assume that $\theta_{\mathcal{O}_{Y}, K}: f^{!} \mathcal{O}_{Y} \otimes^{\mathrm{L}}$ $\mathrm{L} f^{*} K \rightarrow f^{!} K$ is an isomorphism. Then we can rewrite the push-forward of Theorem 4.4 as

$$
f_{*}: \mathrm{W}^{i+d}\left(X, \omega_{f} \otimes^{\mathrm{L}} \mathrm{L} f^{*} K\right) \rightarrow \mathrm{W}^{i}(Y, K) .
$$

In particular, the hypotheses and therefore the conclusion hold if $K$ is dualizing and either of the two following conditions hold.

- $f$ is quasi-perfect (see below, e.g. of finite tor-dimension) and $f^{!} K$ is dualizing.

- $Y$ is a Gorenstein scheme, e.g. regular.

Proof. The reformulation of the push-forward is Definitions 6.1.3 and 6.1.4, [12]. When $f$ is quasi-perfect, Proposition 2.1 in [27] shows that $\theta$ is an isomorphism on all $D_{\mathrm{qc}}(Y)$. Example 2.2 in loc. cit. shows that if $f$ is of finite tor-dimension, it is quasi-perfect. When $Y$ is Gorenstein, the only dualizing complexes are shifted line bundles, for which $\theta$ is an isomorphism by Lemma 5.6.

Let $g: Y \rightarrow Z$ be another proper morphisms in $\S c h$ and $M$ a dualizing complex on $Z$ and let

$$
\iota_{f, g}: \omega_{f} \otimes{ }^{\mathrm{L}} \mathrm{L} f^{*}\left(\omega_{g} \otimes^{\mathrm{L}} \mathrm{L}_{g}^{*} M\right) \rightarrow \omega_{g f} \otimes^{\mathrm{L}} \mathrm{L}(g f)^{*} M
$$

be the morphism defined in Theorem 6.1.5 of [12].

Theorem 6.3. The push-forward of Theorem 6.2 respects composition: the morphism $\iota_{f, g}$ is an isomorphism and if I denotes the isomorphism of Witt groups induced by $\iota_{f, g}$, then the push-forward on Witt groups defined above satisfies that $g_{*} f_{*}=(g f)_{*} I$.

Proof. This follows from Theorem 6.1.5 and Lemma 2.2.6(2) in [12]. Note that $\iota_{f, g}$ is an isomorphism because it is a composition of isomorphisms under the assumptions for the reformulated push-forward to exist. 
Theorem 6.4. In the situation of Theorem 5.5 and under the assumptions of the reformulation of the push-forward above for the morphisms $g$ and $\bar{g}$, the base change theorem 5.5 becomes

$$
f^{*} g_{*}=\bar{g}_{*} I \bar{f}_{*}
$$

where I is the isomorphism of Witt groups induced by the isomorphism

$$
\iota: \mathrm{L} \bar{f}^{*}\left(\omega_{g} \otimes^{\mathrm{L}} \mathrm{L} g^{*} K\right) \rightarrow \omega_{\bar{g}} \otimes^{\mathrm{L}} \mathrm{L}^{*} \mathrm{~L} f^{*} K .
$$

Proof. This follows from Theorem 6.1.7 in [12]. Note that $\gamma_{\mathcal{O}_{Z}}$ is an isomorphism (see before Theorem 5.5).

In the regular case, the projection formula 5.7 becomes the following.

Theorem 6.5. Let $f: X \rightarrow Y$ be a proper equidimensional morphism of relative dimension $d$ with $X, Y \in \mathcal{R e g}$. Then the push-forward of Theorem 6.2 and the pullback of Theorem 4.1 satisfy $f_{*} I\left(x . f^{*}(y)\right)=f_{*}(x) \cdot y$ in $\mathrm{W}^{i+j}(Y, L \otimes K)$ for any $x \in \mathrm{W}^{i+d}\left(X, \omega_{f} \otimes f^{*} L\right)$ and $y \in \mathrm{W}^{j}(Y, K)$.

Proof. See [12], Theorem 6.1.9 and Corollary 6.1.10.

6.2. Smooth schemes over a base. We now fix a base scheme $S \in \delta c h$ with a dualizing complex $K_{S}$ and consider the category $S m P r / S$ of schemes in $S c h$ that are smooth, equidimensional and proper over $S$. For such a scheme $X$, let the structural morphism be denoted by $p_{X}: X \rightarrow S$ and its relative dimension over $S$ by $d_{X}$. Note that any separated morphism between schemes in $\delta m \operatorname{Pr} / S$ is proper, being the composition of a closed embedding, its graph, and a proper projection.

Notation 6.6. Let $X \in S m P r / S$. We set $\omega_{X}=p_{X}^{!}\left(K_{S}\right)\left[-d_{X}\right]$. Observe that $\omega_{X}=\omega_{p_{X}}$ if $K_{S}=\mathcal{O}_{S}$.

Theorem 6.7. Let $f: X \rightarrow Y$ be a separated morphism, $X, Y \in \operatorname{SmPr} / S$ and let $L$ be a line bundle on $Y$. The push-forward can be written

$$
f_{*}: \mathrm{W}^{i+d_{X}}\left(X, \omega_{X} \otimes f^{*} L\right) \rightarrow \mathrm{W}^{i+d_{Y}}\left(Y, \omega_{Y} \otimes L\right)
$$

Proof. First, let us note that when pulling back or tensoring by a line bundle, there is nothing to derive. This is why no $\mathrm{L}$ appear in front of $f^{*}$ and $\otimes$. We then use the definitions in [12] (Definitions 6.3.3 and 6.3.4). We need to check that the morphism

$$
\omega_{X} \otimes L \simeq f^{!} \omega_{Y} \otimes f^{*} L \rightarrow f^{!}\left(\omega_{Y} \otimes L\right)
$$

is an isomorphism. This is the case by Lemma 5.6. 
Theorem 6.8. The push-forward of Theorem 6.7 respects composition.

Proof. See [12], Theorem 6.3.5 and Corollary 6.3.6.

Theorem 6.9. The push-forward of Theorem 6.7 satisfies flat base change.

Proof. See [12], Theorem 6.3.7 and Corollary 6.3.8.

\section{Examples}

Note that $f^{!}$is unique up to unique isomorphism whenever it is defined, because it is always defined as a right adjoint to $\mathrm{R} f_{*}$. This allows us to use computations of $f$ ! from [23] and other sources in the examples below.

7.1. Finite field extensions. The simplest example of a proper morphism is the case of a finite field extension $E / F$ giving rise to a finite morphism

$$
f: X=\operatorname{Spec}(E) \rightarrow \operatorname{Spec}(F)=Y .
$$

The tilde construction gives equivalences of categories $\operatorname{Mod}(F) \simeq \mathrm{Q} \operatorname{coh}(Y)$ and $\operatorname{Mod}(E) \simeq \mathrm{Q} \operatorname{coh}(X)$, and the subcategories of finite dimensional vector spaces correspond to coherent sheaves of modules. We thus describe all objects and functors through these equivalences of categories. The only dualizing complex (up to shifts and isomorphisms) on $Y$ is $F$ itself. The functors $f^{*}=\left(-\otimes_{F} E\right)$ and $f_{*}=\left.(-)\right|_{F}$ are exact, there is nothing to derive. The functor $f^{!}$is given by III, §6, of [23] as $\mathcal{H o m}_{F}(E,-)$ (mapping to $E$-vector spaces) and the unit and counit of the adjunction $\left(f_{*}, f^{!}\right)$are respectively given by

$$
\begin{aligned}
V & \rightarrow \mathcal{H}_{F}\left(E,\left.V\right|_{F}\right), & \left.\mathscr{H o m}_{F}\left(E, V^{\prime}\right)\right|_{F} & \rightarrow V^{\prime}, \\
a & \mapsto(e \mapsto e . a), & \phi & \mapsto \phi(1),
\end{aligned}
$$

for an $E$-vector space $V$ and an $F$-vector space $V^{\prime}$. For fields, the only nonzero Witt group modulo 4 is $\mathrm{W}^{0}$ which is the classical Witt group of the field. So we are reduced to study push-forward for forms on vector spaces, i.e. complexes concentrated in degree zero. Following the construction, it is easy to check that for any $E$-vector space $V$, the morphism $\zeta: f_{*}\left[V, f^{!} F\right] \rightarrow\left[f_{*} V, F\right]$ coincides with the Cartan isomorphism of $F$-vector spaces

$$
\left.\mathscr{H o m}_{E}\left(V, \mathscr{H o m}_{F}(E, F)\right)\right|_{F} \simeq \mathscr{H o m}_{F}\left(\left.V\right|_{F}, F\right)
$$

which sends a morphism $\phi: V \rightarrow \mathscr{H}_{F}(E, F)$ to the morphism $(a \mapsto \phi(a)(1))$. Thus, the push-forward $f_{*}: \mathrm{W}^{0}\left(E, \mathcal{H} \mathrm{om}_{F}(E, F)\right) \rightarrow \mathrm{W}^{0}(F)$ is a Scharlau transfer 
(see [34], p. 48) with respect to the usual trace $\operatorname{Tr}: E \rightarrow F$. To see this, note that $\operatorname{Tr}$ factors as $E \stackrel{\simeq}{\rightarrow} \mathscr{H}_{\mathrm{om}_{F}}(E, F) \rightarrow F$ where the isomorphism is given by $e \mapsto(x \mapsto \operatorname{Tr}(e . x))$ and $\operatorname{Hom}_{F}(E, F) \rightarrow F$ is the evaluation at 1 .

7.2. Regular embeddings. Let $F$ be a vector bundle of rank $d>0$ over $X$ with a regular section $s: \mathcal{O}_{X} \rightarrow F$, i.e. such that the corresponding embedding $f: Z \subset X$ of the zero locus is a closed regular embedding of codimension $d$. In this case the augmented Koszul resolution is exact, see Proposition 3.1 (IV, §2) in [14], and thus yields a quasi-isomorphism

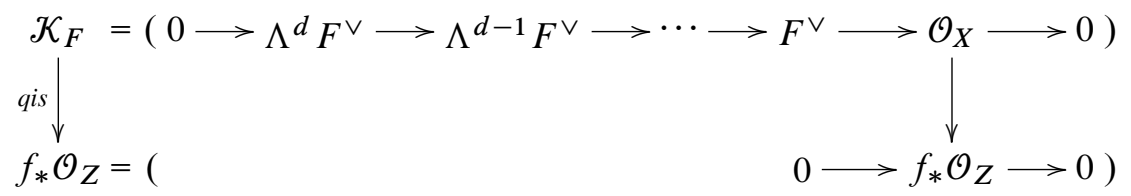

from the Koszul complex $\mathcal{K}_{F}$ to $f_{*} \mathcal{O}_{Z}$ concentrated in degree 0 . Since $f$ is a closed embedding, thus finite, $f_{*}$ is exact and coincides with $\mathrm{R} f_{*}$. Let $\Delta_{F}=\Lambda^{d} F$ be the determinant of $F$. In this situation, we have $f^{!} A=f^{*} \Delta_{F}[-d] \otimes \mathrm{L} f^{*} A$ for all $A \in D_{\mathrm{b}, \mathrm{c}}(X)$; this may be extracted from III, §7, [23], see also Proposition 1 in [37], after applying Lemma 5.6 and using that $F$ is dual to the cotangent sheaf. By tensoring the augmented Koszul resolution with $\Delta_{F}$ and using the canonical isomorphisms $\Lambda^{i} F^{\vee} \otimes \Delta_{F} \cong \Lambda^{d-i} F$ and $f_{*} \mathcal{O}_{Z} \otimes \Delta_{F} \cong f_{*} f^{*} \Delta_{F}$, we obtain the trace map $f_{*} f^{!} \mathcal{O}_{X} \rightarrow \mathcal{O}_{X}$ (counit of the adjunction $\left(\mathrm{R} f_{*}, f^{!}\right)$) in the derived category as the composition of a usual map of complexes followed by the inverse of a quasi-isomorphism $\left(\mathcal{O}_{X}\right.$ is in degree 0 and $f_{*} f^{*} \Delta_{F}$ in degree $\left.-d\right)$ :

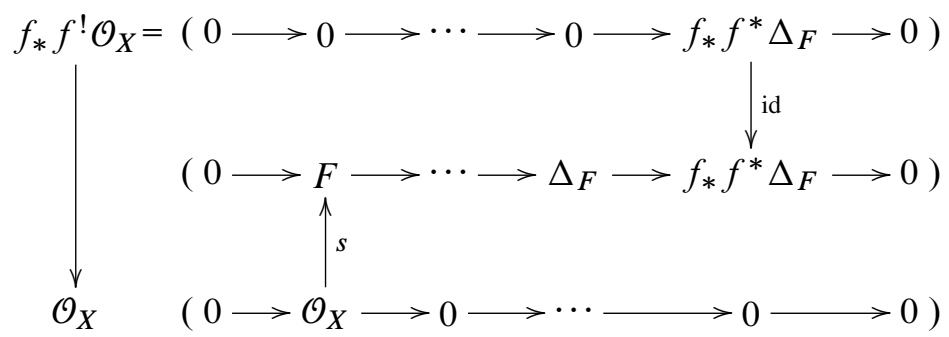

Now assume $Z$ is Gorenstein. Then $\mathcal{O}_{Z}$ is dualizing, and the isomorphism $\mathcal{O}_{Z} \rightarrow$ $\left[\mathcal{O}_{Z}, \mathcal{O}_{Z}\right]$ adjoint to $\mathcal{O}_{Z} \otimes^{\mathrm{L}} \mathcal{O}_{Z} \simeq \mathcal{O}_{Z}$ defines a form on $\mathcal{O}_{Z}$, denoted by $\mathbf{1}_{Z}$. On the other hand, there is a well-known form $\theta_{F}: \mathcal{K}_{F} \rightarrow \mathscr{H}^{\circ} \mathrm{m}\left(\mathcal{K}_{F}, \Delta_{F}^{\vee}[d]\right.$ ) (see $\S 4$ in [6]) given by the canonical isomorphism $\Lambda^{i} F^{\vee} \simeq\left(\Lambda^{d-i} F^{\vee}\right)^{\vee} \otimes \Lambda^{d} F^{\vee}$ in degree $i$, with a sign chosen so that when $F=\oplus L_{i}$ is a direct sum of line bundles, this 
form is the tensor product of forms $\theta_{L_{i}}$ on Koszul complexes of length one

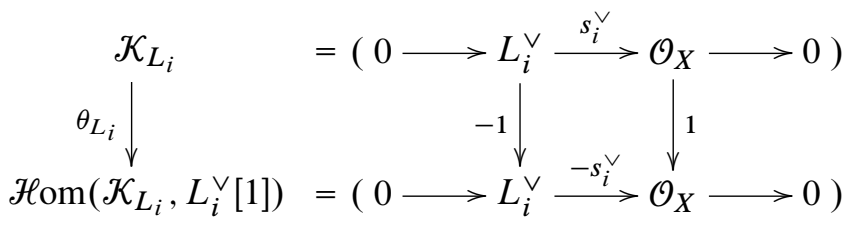

representing elements in $\mathrm{W}^{1}\left(X, L_{i}^{\vee}\right)$. The following proposition can be considered as a concrete description of the push-forward of $\mathbf{1}_{Z}$ along $f$.

Proposition 7.1. Let $Z$ and $X$ be Gorenstein schemes and $f: Z \rightarrow X$ be a closed regular embedding of codimension d defined as the zero locus of a regular section of a vector bundle $F$ of rank $d$ whose determinant $\Lambda^{d} F$ is denoted by $\Delta_{F}$. Then the image of the form $\mathbf{1}_{Z}$ : $\mathcal{O}_{Z} \stackrel{\simeq}{\rightarrow}\left[\mathcal{O}_{Z}, \mathcal{O}_{Z}\right]$ (adjoint to $\mathcal{O}_{Z} \otimes^{\mathrm{L}} \mathcal{O}_{Z} \simeq \mathcal{O}_{Z}$ ) under the composition

$$
\mathrm{W}^{0}\left(Z, \mathcal{O}_{Z}\right) \simeq \mathrm{W}^{d}\left(Z, f^{!} \Delta_{F}^{\vee}\right) \stackrel{f_{*}}{\longrightarrow} \mathrm{W}^{d}\left(X, \Delta_{F}^{\vee}\right)
$$

is a form $\phi$ such that the following diagram in $D(X)$ commutes.

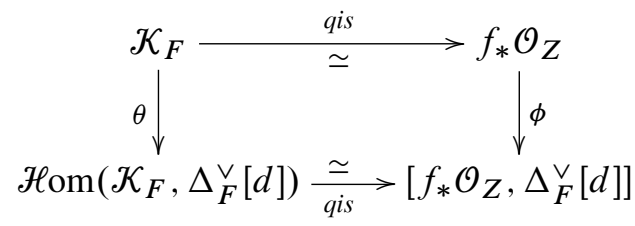

Proof. Let $\delta_{F}: \mathcal{K}_{F} \otimes \mathcal{K}_{F} \rightarrow \mathcal{K}_{F}$ in $D(X)$ be the composition

$$
\mathcal{K}_{F} \otimes \mathcal{K}_{F} \stackrel{\text { qis } \otimes i s}{\longrightarrow} f_{*} \mathcal{O}_{Z} \otimes^{\mathrm{L}} f_{*} \mathcal{O}_{Z} \stackrel{\lambda}{\longrightarrow} f_{*}\left(\mathcal{O}_{Z} \otimes^{\mathrm{L}} \mathcal{O}_{Z}\right) \stackrel{\simeq}{\longrightarrow} f_{*} \mathcal{O}_{Z} \stackrel{q i s^{-1}}{\longrightarrow} \mathcal{K}_{F}
$$

where $\lambda$ is the morphism from Proposition 4.2.1 in [12]. Note that $\delta_{F}$ is in fact represented by a morphism of complexes (not just a fraction): one can check that the map from $\mathcal{K}_{F} \otimes \mathcal{K}_{F}$ to $\mathcal{K}_{F}$ in degree $i$ is a sum of the canonical morphisms $\Lambda^{k} F \otimes$ $\Lambda^{i-k} F \rightarrow \Lambda^{i} F$ with appropriate signs. We also consider the map $\sigma_{F}: \mathcal{K}_{F} \rightarrow$ $\Delta_{F}^{\vee}[d]$ given by

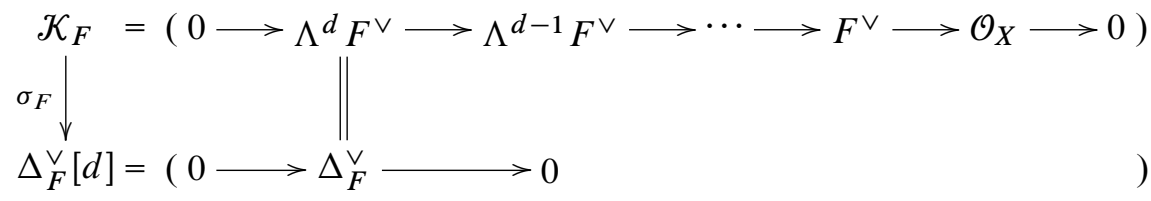

The following three lemmas together clearly imply the proposition. 
Lemma 7.2. The morphism of complexes $x_{F}: \mathcal{K}_{F} \rightarrow \mathcal{H} \mathrm{om}\left(\mathcal{K}_{F}, \Delta_{F}^{\vee}[d]\right)$ defined as the composition

$$
\mathcal{K}_{F} \rightarrow \mathscr{H}_{\mathrm{om}}\left(\mathcal{K}_{F}, \mathcal{K}_{F} \otimes \mathcal{K}_{F}\right) \stackrel{\left(\delta_{F}\right)_{*}}{\longrightarrow} \mathcal{H o m}\left(\mathcal{K}_{F}, \mathcal{K}_{F}\right) \stackrel{\left(\sigma_{F}\right)_{*}}{\longrightarrow} \mathcal{H o m}\left(\mathcal{K}_{F}, \Delta_{F}^{\vee}[d]\right)
$$

coincides with the form $\theta$ where the first map is the unit of the adjunction of the tensor product and the internal Hom in the homotopy category.

Lemma 7.3. The form $\phi: f_{*} \mathcal{O}_{Z} \rightarrow\left[f_{*} \mathcal{O}_{Z}, \Delta_{F}^{\vee}[d]\right]$ coincides with the composition

$$
\begin{aligned}
& f_{*} \mathcal{O}_{Z} \longrightarrow\left[f_{*} \mathcal{O}_{Z}, f_{*} \mathcal{O}_{Z} \otimes^{\mathrm{L}} f_{*} \mathcal{O}_{Z}\right] \stackrel{\lambda}{\longrightarrow}\left[f_{*} \mathcal{O}_{Z}, f_{*}\left(\mathcal{O}_{Z} \otimes^{\mathrm{L}} \mathcal{O}_{Z}\right)\right] \\
& {\left[f_{*} \mathcal{O}_{Z}, \Delta_{F}^{\vee}[d]\right] \longleftarrow\left[f_{*} \mathcal{O}_{Z}, f_{*} f^{!} \Delta_{F}^{\vee}[d]\right] \longleftarrow \simeq\left[f_{*} \mathcal{O}_{Z}, f_{*} \mathcal{O}_{Z}\right]}
\end{aligned}
$$

where the first map is the unit of the adjunction of the tensor product and the internal Hom in $D(X)$, the penultimate one is the identification $\mathcal{O}_{Z} \simeq f^{!} \Delta_{F}^{\vee}[d]$ and the last one is induced by the counit of the adjunction $\left(f_{*}, f^{!}\right)$, i.e. the trace map described above.

Lemma 7.4. The composition of Lemma 7.2 coincides with the one of Lemma 7.3 when $f_{*} \mathcal{O}_{Z}$ is identified with $\mathcal{K}_{F}$ using qis.

Proof of Lemma 7.2. As we are dealing with honest morphisms of complexes we may first reduce to open subsets on which $F$ is a sum of line bundles $L_{i}$ (note that two morphisms in $D(X)$ are not necessarily equal if they are equal when restricted to all open sets of an affine covering, see for example [5]). We then reduce to the case of codimension $d=1$, by multiplicativity of Koszul complexes: let $d=d_{1}+d_{2}$ and let $F=F_{1} \oplus F_{2}$ where $F_{1}$ (resp. $F_{2}$ ) is the sum of the first $d_{1}$ line bundles (resp. last $d_{2}$ ). Let $f_{1}: \mathcal{O}_{Z_{1}} \rightarrow X$ (resp. $f_{2}: \mathcal{O}_{Z_{2}} \rightarrow X$ ) be the corresponding regular subschemes. Then $\Delta_{F} \simeq \Delta_{F_{1}} \otimes \Delta_{F_{2}}$ and $\mathcal{K}_{F} \simeq \mathcal{K}_{F_{1}} \otimes \mathcal{K}_{F_{2}}$. We leave it to the reader to show that the diagram

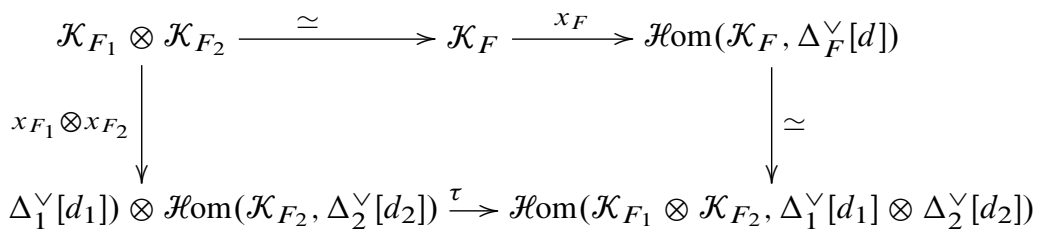

commutes, where $\tau$ is the morphism defined as in [12] (Definition 4.4.1), using the monoidal structure on the homotopy category. By definition, $\theta_{F}, \theta_{F_{1}}$ and $\theta_{F_{2}}$ make the same diagram commutative when they replace $x_{F}, x_{F_{1}}$ and $x_{F_{2}}$. Hence it suffices to show the lemma for one line bundle $L$ and its associated Koszul complex of length one, which can be checked by hand. 
Proof of Lemma 7.3. By definition, the form $\phi$ is given by the composition

$$
f_{*} \mathcal{O}_{Z} \stackrel{f_{*} \mathbf{1}_{Z}}{\longrightarrow} f_{*}\left[\mathcal{O}_{Z}, \mathcal{O}_{Z}\right] \stackrel{\simeq}{\longrightarrow} f_{*}\left[\mathcal{O}_{Z}, f^{!} \Delta_{F}^{\vee}[d]\right] \stackrel{\zeta}{\longrightarrow}\left[f_{*} \mathcal{O}_{Z}, \Delta_{F}^{\vee}[d]\right] .
$$

One proves using the closed monoidal structure that it coincides with the composition

$$
f_{*} \mathcal{O}_{Z} \stackrel{\simeq}{\longrightarrow} f_{*} f^{!} \Delta_{F}^{\vee}[d] \longrightarrow f_{*}\left[\mathcal{O}_{Z}, f^{!} \Delta_{F}^{\vee}[d]\right] \stackrel{\zeta}{\longrightarrow}\left[f_{*} \mathcal{O}_{Z}, \Delta_{F}^{\vee}[d]\right]
$$

where the second map is adjoint to the unit morphism of the monoidal structure. Then, looking back at the definition of $\zeta$ and $\mu$ in Proposition 4.2.2 and Theorem 4.2.9 of [12], one sees that $\phi$ is the composition around the lower left corner of the commutative diagram

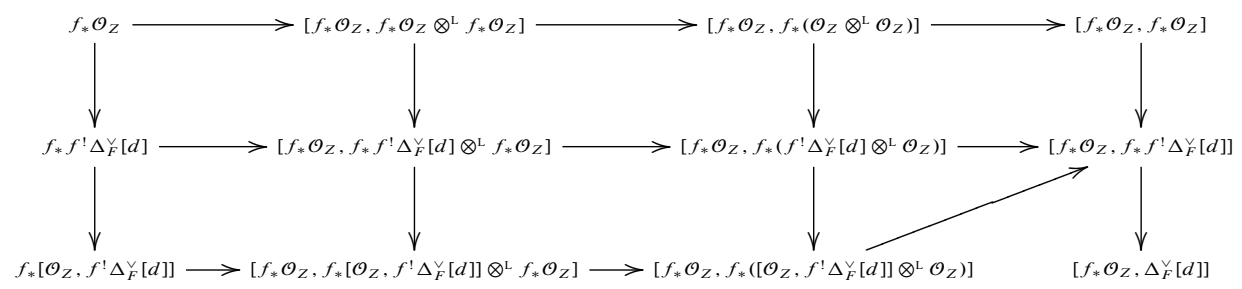

which thus proves the lemma (all squares in this diagram are commutative by obvious functorial reasons, and the triangle by adjunction).

Proof of Lemma 7.4. This follows from the computation of the resolution of $f_{*} \mathcal{O}_{Z}$ by $\mathcal{K}_{F}$ when computing the derived functors $\left[f_{*} \mathcal{O}_{Z},-\right]$ and $-\otimes^{\mathrm{L}} f_{*} \mathcal{O}_{Z}$.

This finishes the proof of Proposition 7.1.

Remark 7.5. If $F=F^{\prime} \oplus L_{1}$, with $L_{1}$ a line bundle, $s=\left(s^{\prime}, s_{1}\right), s^{\prime}$ and $s_{1}$ transverse, the push-forward of $\mathbf{1}_{Z}$ is zero: decompose the inclusion $Z \subset X$ as $Z \subset Z\left(s^{\prime}\right) \subset X$ where $Z\left(s^{\prime}\right)$ is the zero locus of $s^{\prime}$. Push-forwards respect composition and the pushforward of $\mathbf{1}_{Z}$ along $Z \subset Z\left(s^{\prime}\right)$ is already zero since it is the form (4) which is the cone of a (degenerate) form $s: L_{1}^{\vee} \rightarrow \mathcal{O}_{X}$.

On the other hand, an example when this push-forward is nonzero can be extracted from [4]. Let $k$ be a field and let $\operatorname{Gr}_{k}(2,4)$ be the Grassmannian of 2-planes in $\mathbb{A}_{k}^{4}$. A nonzero map from $\mathbb{A}_{k}^{4}$ to $\mathbb{A}_{k}$ induces a section of the dual $W^{\vee}$ of the universal subbundle $W \subset \mathbb{A}_{k}^{4}$ of rank 2 . Its zero locus is a copy of $\mathbb{P}^{2}$ regularly embedded in $\operatorname{Gr}(2,4)$. The push-forward of the unit form of $\mathbb{P}^{2}$ to $\operatorname{Gr}(2,4)$ is nonzero by [4] where it is proved that it is an element of a basis of the total Witt group of $\operatorname{Gr}(2,4)$ as a $\mathrm{W}(k)$-module. 
7.3. Projective spaces. Let $Y \in \delta c h$ be a Gorenstein scheme, let $\mathcal{E}$ be a vector bundle of rank $r+1$ on $Y$ and let us examine when the unit form on $X=\mathbb{P}_{Y}(\mathcal{E})$ can be pushed forward to $Y$ along $f: X \rightarrow Y$. Since $f$ is smooth (thus flat), we can use Section 6.1. In the case of a smooth morphism $f$, the object $\omega_{f}$ of 6.1 is a line bundle, and it is the maximal exterior power of the relative cotangent bundle (see Chapter VII $\S 4$ in [23]). Here, since $f$ is a projective bundle, it is given by $\omega_{f}=f^{*}\left(\Delta_{\mathcal{E}}\right)^{\vee} \otimes \mathcal{O}(-r-1)$ (see e.g. [15], Appendix B.5.8). If $r+1$ is even, we can push-forward the unit form $\mathbf{1}_{X}: \mathcal{O}_{X} \simeq\left[\mathcal{O}_{X}, \mathcal{O}_{X}\right]$ from $\mathrm{W}^{0}\left(X, \mathcal{O}_{X}\right)$ by using the composition

$$
\mathrm{W}^{0}\left(X, \mathcal{O}_{X}\right) \simeq \mathrm{W}^{0}(X, \mathcal{O}(-r-1))=\mathrm{W}^{0}\left(X, \omega_{f} \otimes f^{*}\left(\Delta_{\mathcal{E}}\right)\right) \rightarrow \mathrm{W}^{-r}\left(Y, \Delta_{\mathcal{E}}\right)
$$

where the first isomorphism is given by tensoring with the canonical form $\phi_{r}=$ $\left[\mathcal{O}(-(r+1) / 2) \stackrel{\simeq}{\rightarrow} \mathcal{O}((r+1) / 2) \otimes \mathcal{O}(-(r+1)) \cong \mathcal{H o m}_{\mathcal{O}}(\mathcal{O}(-(r+1) / 2), \mathcal{O}(-(r+\right.$ $1))]$ and the last map is the push-forward in the form of Theorem 6.2. Computing the image of $\mathbf{1}_{X}$ through this composition means therefore computing the image of $\phi_{r}$ by the push-forward. The complex on which $f_{*}\left(\phi_{r}\right)$ lives is $\mathrm{R} f_{*}(\mathcal{O}(-(r+1) / 2))$. But this complex is zero by [20], 2.1.15, so $f_{*}\left(\phi_{r}\right)=0$. If $r+1$ is odd, there is no push-forward induced by $f$ with source $\mathrm{W}^{0}\left(X, \mathcal{O}_{X}\right)$ because then there is no line bundle $K$ on $Y$ such that $\mathcal{O}_{X}$ is equal to $f^{*}\left(\Delta_{\mathcal{E}}^{\vee}\right) \otimes \mathcal{O}(-r-1) \otimes f^{*}(K)$ up to a square in $\operatorname{Pic}(Y)$. In other words, pushing forward the unit form of $\mathbb{P}_{Y}(\mathcal{E})$ to $Y$ is not very interesting: whenever it is possible, we get zero. Of course, there are other forms on $\mathbb{P}^{r}(\mathscr{E})$ not mapping to 0 under the push-forward, as we will see in the following remark.

Remark 7.6. Let us explain a potential source of confusion. Let $i: \operatorname{Spec} k \rightarrow \mathbb{P}_{k}^{r}$ be a rational point and $L$ a line bundle on $\mathbb{P}_{k}^{r}$. Since $\operatorname{Pic}(\operatorname{Spec} k)=0$, using first an isomorphism $\mathcal{O}_{k} \simeq \omega_{i} \otimes i^{*}(L)$, we can push-forward from $\mathrm{W}^{0}\left(\operatorname{Spec} k, \mathcal{O}_{k}\right)$ to $\mathrm{W}^{r}\left(\mathbb{P}_{k}^{r}, L\right)$ for any $L$. But for different $L$, we get very different push-forwards. Indeed, for example $\mathrm{W}^{r}\left(\mathbb{P}_{k}^{r}, \mathcal{O}(-r)\right)=0$ for odd $r$ (by [39] or [3]) so any pushforward to there is obviously zero, whereas since $\omega_{k} \simeq \mathcal{O}_{k}$ the push-forward (written as in Theorem 6.7)

$$
\mathrm{W}^{0}\left(\operatorname{Spec} k, \mathcal{O}_{k}\right) \simeq \mathrm{W}^{0}\left(\operatorname{Spec} k, \omega_{k}\right) \rightarrow \mathrm{W}^{r}\left(\mathbb{P}_{k}^{r}, \omega_{\mathbb{P}_{k}^{r}}\right)
$$

is certainly nonzero, because we can further compose it by a push-forward back to $\mathrm{W}^{0}\left(\operatorname{Spec} k, \omega_{k}\right)$ and since the push-forward respects composition, the composite is the identity. Note that this last case also gives an example of a form on $\mathbb{P}_{k}^{r}$ whose pushforward to Spec $k$ is not zero. More generally, this phenomenon of different pushforwards starting from the same group can happen whenever $f^{*}: \operatorname{Pic}(Y) \rightarrow \operatorname{Pic}(X)$ is not injective. 


\section{A. q-flat and q-injective resolutions}

For the convenience of the reader, we include here well-known facts on q-flat or qinjective objects that are repeatedly used in the proofs of this article. Most of them are due to Spaltenstein.

Definition A.1. Let $X$ be a scheme and let $A$ be an object in the homotopy category $K(X)$. We say that $A$ is q-flat (or $\mathrm{K}$-flat) if the triangulated functor $(-\otimes A): K(X) \rightarrow K(X)$ preserves quasi-isomorphisms. We say that $A$ is qinjective (or K-injective) if the triangulated functor $\mathscr{H}_{\bullet}(-, A): K(X)^{o} \rightarrow K(X)$ preserves quasi-isomorphisms.

Example A.2. A bounded above complex of flat $\mathcal{O}_{X}$-modules is q-flat. A bounded below complex of injectives is q-injective.

A discussion of q-flat and q-injective complexes can be found in Sections 1 and 5 of [35]. See in particular Propositions 1.5 and 5.3.

Proposition A.3. Let $A$ and $B$ be objects in $K(X)$ or $K(Y)$ and let $f: X \rightarrow Y$ be a morphism of schemes.

(1) If $A$ and $B$ are $q$-flat, then so is $A \otimes B$.

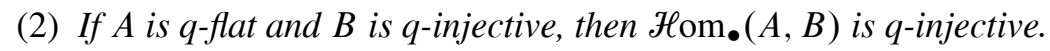

(3) If $A$ is q-flat, then $f^{*} A$ is q-flat.

Proof. See [35], Proposition 5.3 and 5.4.

The following two propositions summarize the equivalences of categories and the properties of injectives that we need. Let $\mathrm{Q} \operatorname{coh}(X)$ denote the abelian category of quasi-coherent sheaves on $X$ and $\mathrm{C}_{\mathrm{b}}(\operatorname{Vect}(X))\left(\operatorname{resp} . \mathrm{C}_{\mathrm{b}}(\operatorname{Coh}(X))\right)$ the category of bounded complexes of locally free (resp. coherent) sheaves on $X$.

Proposition A.4. Let $X \in S c h$.

(1) The natural functor $D(\mathrm{Q} \operatorname{coh}(X)) \rightarrow D_{\mathrm{qc}}(X)$ is an equivalence of categories and thus the same is true for their homologically bounded, bounded below or bounded above subcategories and the subcategories with coherent homology.

(2) The natural functor $D_{\mathrm{b}}(\operatorname{Coh}(X)) \rightarrow D_{\mathrm{b}, \mathrm{c}}(\mathrm{Q} \operatorname{coh}(X))$ is an equivalence.

(3) If $X \in \mathcal{R e g}$, then the natural functor $D\left(\mathrm{C}_{\mathrm{b}}(\operatorname{Vect}(X))\right) \rightarrow D_{\mathrm{b}, \mathrm{c}}(X)$ is an equivalence of categories. 
Proof. Point (1) is Corollary 5.5 in [9]. In Point (2), fully faithful follows from Theorem 12.1 in [25], second part: For affine schemes, use Example 12.3 in [25]. In general, take a finite affine cover of the noetherian scheme $X$ and then take the direct sum over the coherent sheaves on $X$ obtained by extending the coherent sheaves on the affine subschemes using [22], I.6.9.7. Essentially surjective can be found in [17], Example 2.5.2, which follows from Lemme 2.1.2.c) of [38] and an induction argument. Point (3) can then be proved as follows. Let $\mathrm{C}_{\mathrm{b}}(\operatorname{Coh}(X))$ be the category of bounded complexes of coherent sheaves. Decompose the functor $D\left(\mathrm{C}_{\mathrm{b}}(\operatorname{Vect}(X))\right) \rightarrow D_{\mathrm{b}, \mathrm{c}}(X)$ as

$$
\begin{gathered}
D\left(\mathrm{C}_{\mathrm{b}}(\operatorname{Vect}(X))\right) \longrightarrow D\left(\mathrm{C}_{\mathrm{b}}(\operatorname{Coh}(X))\right) \longrightarrow D_{\mathrm{b}}(\operatorname{Coh}(X)) \\
\longrightarrow D_{\mathrm{b}, \mathrm{c}}(\mathrm{Q} \operatorname{coh}(X)) \longrightarrow D_{\mathrm{b}, \mathrm{c}}(X) .
\end{gathered}
$$

All these functors are equivalences of categories: the first one by the fact since $X$ is regular, every coherent sheaf has a finite resolution by locally free sheaves by [33], $\S 7$, Point 1, the second one by Lemma 11.7 of [25], the third one by Point (2) and the fourth by Point (1).

Proposition A.5. Let $X \in S c h$.

(1) The category Qcoh $(X)$ has enough injectives by [36], B.3.

(2) The natural inclusion $\mathrm{Q} \operatorname{coh}(X) \rightarrow \operatorname{Mod}(X)$ preserves injectives by [36], B.4.

(3) Every bounded below complex of quasi-coherent $\mathcal{O}_{X}$-modules admits a quasiisomorphism into a complex of Qcoh $(X)$-injectives by (1) and [23], I.4.6.

(4) Every bounded below complex of Qcoh $(X)$-injectives is q-injective in both $K(\mathrm{Q} \operatorname{coh}(X))$ and $K(X)$ by $(2)$.

Corollary A.6. Let $X \in S$ ch. On objects in $D_{-, \mathrm{qc}}(X)$ or $D_{-}(X)$, the unbounded right derived functors computed by q-injective resolutions (as in [35]) coincide with the more classical bounded below right derived functors computed by using resolutions by bounded below complexes of injectives (as in [23]).

Proof. By the proposition, any object $A \in D_{-, \mathrm{qc}}(X)$ is quasi-isomorphic to a bounded below complex of $\mathrm{Q} c o h(X)$-injectives which are also $\operatorname{Mod}(X)$-injective (resp. a bounded below complex of $\operatorname{Mod}(X)$-injectives) and this complex is q-injective.

Similarly, for q-flat resolutions, we have a weaker statement, sufficient for our purposes.

Proposition A.7. Let $X \in S c h$. On objects in $D_{+, \mathrm{qc}}(X)$ or $D_{+}(X)$, the unbounded left derived functors computed using q-flat resolutions can be computed by using bounded above resolutions consisting of flat $\mathcal{O}_{X}$-modules. 
Proof. This follows from the fact that any $\mathcal{O}_{X}$-module is a quotient of a flat one (see [28], 2.5.5).

\section{References}

[1] L. Alonso Tarrío, A. Jeremías López, and J. Lipman, Local homology and cohomology on schemes. Ann. Sci. École Norm. Sup. (4) 30 (1997), no. 1, 1-39. Zbl 0894.14002 MR 1422312444

[2] P. Balmer, Triangular Witt groups. I. The 12-term localization exact sequence. $K$-Theory 4 (2000), no. 19, 311-363. Zbl 0953.18003 MR 1763933 437, 447

[3] P. Balmer, Products of degenerate quadratic forms. Compos. Math. 141 (2005), no. 6, 1374-1404. Zbl 1087.18008 MR 2188441463

[4] P. Balmer and B. Calmès, Witt groups of Grassmann varieties. Preprint 2008. arXiv:0807.3296 438, 462

[5] P. Balmer and G. Favi, Gluing techniques in triangular geometry. Q. J. Math. 58 (2007), no. 4, 415-441. Zbl 1130.18006 MR 2371464461

[6] P. Balmer and S. Gille, Koszul complexes and symmetric forms over the punctured affine space. Proc. London Math. Soc. (3) 91 (2005), no. 2, 273-299. Zbl 1078.11026 MR 2167088459

[7] A. A. Beřlinson, Coherent sheaves on $\mathbf{P}^{n}$ and problems in linear algebra. Funktsional. Anal. i Prilozhen. 12 (1978), no. 3, 68-69. Zbl 0424.14003 MR 0509388450

[8] P. Berthelot, A. Grothendieck et L. Illusie (eds.), Théorie des intersections et théorème de Riemann-Roch. Séminaire de Géométrie Algégrique du Bois-Marie 1966-1967 (SGA 6), avec la collaboration de D. Ferrand, J. P. Jouanolou, O. Jussila, S. Kleiman, M. Raynaud et J. P. Serre, Lecture Notes in Math. 225, Springer-Verlag, Berlin 1971. Zbl 0218.14001 MR 0354655444

[9] M. Bökstedt and A. Neeman, Homotopy limits in triangulated categories. Compositio Math. 86 (1993), no. 2, 209-234. Zbl 0802.18008 MR 1214458465

[10] B. Calmès and J. Hornbostel, Witt Motives, tranfers and reductive groups. Preprint 2004. http://www.mathematik.uni-bielefeld.de/LAG/ 438, 442

[11] B. Calmès and J. Hornbostel, Witt motives, transfers and dévissage. Preprint 2006. http://www.math.uiuc.edu/K-theory/0786/ 438, 442

[12] B. Calmès and J. Hornbostel, Tensor-triangulated categories and dualities. Theory Appl. Categ. 22 (2009), No. 6, 136-200. Zbl 1178.18005 MR 2520968 438, 439, 440, 441, 442, 444, 445, 446, 447, 448, 449, 450, 451, 452, 453, 454, 455, 456, 457, 458, 460, 461, 462

[13] B. Conrad, Grothendieck duality and base change. Lecture Notes in Math. 1750, SpringerVerlag, Berlin 2000. Zbl 0992.14001 MR 1804902447

[14] W. Fulton and S. Lang, Riemann-Roch algebra. Grundlehren Math. Wiss. 277, SpringerVerlag, New York 1985. Zbl 0885.14002 MR 1644323459

[15] W. Fulton, Intersection theory. 2. ed., Ergeb. Math. Grenzgeb. (3) 2, Springer-Verlag, Berlin 1998. Zbl 0579.14011 MR 0801033463 
[16] S. Gille, A transfer morphism for Witt groups. J. Reine Angew. Math. 564 (2003), 215-233. Zbl 1050.11046 MR 2021041 438

[17] S. Gille, On Witt groups with support. Ph.D. thesis, Wilhelms-Universität Münster, 2001. http://www.math.uni-muenster.de/sfb/about/publ/gille.html 465

[18] S. Gille and A. Nenashev, Pairings in triangular Witt theory. J. Algebra 261 (2003), no. 2, 292-309. Zbl 1016.18007 MR 1966631 438, 455

[19] A. Grothendieck, Éléments de géométrie algébrique. I. Le langage des schémas. Inst. Hautes Études Sci. Publ. Math. 4 (1960), 5-228. Zbl 0118.36206 444, 449

[20] A. Grothendieck, Éléments de géométrie algébrique. III. Étude cohomologique des faisceaux cohérents. I. Inst. Hautes Études Sci. Publ. Math. 11 (1961), 5-167. Zbl 0118.36206 463

[21] A. Grothendieck, Éléments de géométrie algébrique. III. Étude cohomologique des faisceaux cohérents. II. Inst. Hautes Études Sci. Publ. Math. 17 (1963), 5-91. Zbl 0122.16102 MR 0163911449

[22] A. Grothendieck, Eléments de géométrie algébrique I. le langage des schémas. 2. ed., Grundlehren Math. Wiss. 166, Springer-Verlag, Berlin 1971. Zbl 0203.23301 449, 465

[23] R. Hartshorne, Residues and duality. Lecture notes of a seminar on the work of A. Grothendieck, given at Harvard 1963/64. With an appendix by P. Deligne. Lecture Notes in Math. 20, Springer-Verlag, Berlin 1966. Zbl 0212.26101 MR 0222093 444, 446, 447, 448, $450,458,459,463,465$

[24] J. Hornbostel and S. Yagunov, Rigidity for Henselian local rings and $\mathbb{A}^{1}$-representable theories. Math. Z. 255 (2007), no. 2, 437-449. Zbl 1190.14010 MR 2262740438

[25] B. Keller, Derived categories and their uses. In Handbook of algebra, Vol. 1, North-Holland, Amsterdam 1996, 671-701. Zbl 0862.18001 MR 1421815465

[26] G. M. Kelly and S. Maclane, Coherence in closed categories. J. Pure Appl. Algebra 1 (1971), no. 1, 97-140. Zbl 0212.35001 MR 0283045444

[27] J. Lipman and A. Neeman, Quasi-perfect scheme-maps and boundedness of the twisted inverse image functor. Illinois J. Math. 51 (2007), no. 1, 209-236. Zbl 1124.14003 MR 2346195456

[28] J. Lipman, Notes on derived functors and Grothendieck duality. In Foundations of Grothendieck duality for diagrams of schemes, Lecture Notes in Math. 1960, SpringerVerlag, Berlin 2009, 1-259. Zbl 1163.14001 MR 2490557 441, 443, 444, 447, 448, 449, 450, 452, 454, 466

[29] A. Neeman, The Grothendieck duality theorem via Bousfield's techniques and Brown representability. J. Amer. Math. Soc. 9 (1996), no. 1, 205-236. Zbl 0864.14008 MR 1308405 441, 444, 445, 447, 450

[30] A. Neeman, Derived categories and Grothendieck duality. In Triangulated categories, London Math. Soc. Lecture Note Ser. 375, Cambridge University Press, Cambridge 2010, 290-350. Zbl 05831329 MR 2681711 441, 446, 450, 451

[31] A. Nenashev, Gysin maps in Balmer-Witt theory. J. Pure Appl. Algebra 211 (2007), no. 1, 203-221. Zbl 1140.11024 MR 2333767438

[32] A. Nenashev, Projective push-forwards in the witt theory of algebraic varieties. Adv. Math. 220 (2009), no. 6, 1923-1944. Zbl 05541653 MR 2493184438 
[33] D. Quillen, Higher algebraic K-theory: I. In Algebraic K-theory, Lecture Notes in Math. 341, Springer-Verlag, Berlin 1973, 85-147. Zbl 0292.18004 MR 0338129465

[34] W. Scharlau, Quadratic and Hermitian forms, Grundlehren Math. Wiss. 270, SpringerVerlag, Berlin 1985. Zbl 0584.10010 MR 0770063459

[35] N. Spaltenstein, Resolutions of unbounded complexes. Compositio Math. 65 (1988), no. 2, 121-154. Zbl 0636.18006 MR 0932640 441, 443, 444, 448, 449, 452, 464, 465

[36] R. W. Thomason and T. Trobaugh, Higher algebraic K-theory of schemes and of derived categories, In The Grothendieck Festschrift, Vol. III, Progr. Math. 88, Birkhäuser, Boston, Mass., 1990, 247-435. Zbl 0636.18006 MR 1106918 444, 465

[37] J.-L. Verdier, Base change for twisted inverse image of coherent sheaves. In Algebraic Geometry (Internat. Colloq., Tata Inst. Fund. Res., Bombay, 1968), Oxford University Press, London 1969, 393-408. Zbl 0202.19902 MR 0274464 447, 450, 459

[38] J.-L. Verdier, Des catégories dérivées des catégories abéliennes. Astérisque 239 (1996). Zbl 0882.18010 MR 1453167465

[39] C. Walter, Grothendieck-Witt groups of projective bundles. Preprint 2003. http://www.math.uiuc.edu/K-theory/0644/ 438, 463

[40] C. A. Weibel, An introduction to homological algebra. Cambridge Stud. Adv. Math. 38, Cambridge University Press, Cambridge 1994. Zbl 797.18001 MR 1269324447

Received November 14, 2008

Baptiste Calmès, Laboratoire de Mathémathiques de Lens, Faculté des Sciences Jean Perrin, Université d'Artois, Rue Jean Souvraz SP 18, 62307 Lens Cedex, France

E-mail: baptiste.calmes@univ-artois.fr

Jens Hornbostel, Bergische Universität Wuppertal, Fachbereich C / Mathematik und Informatik, Gaußstraße 20, 42119 Wuppertal, Germany

E-mail: hornbostel@math.uni-wuppertal.de 